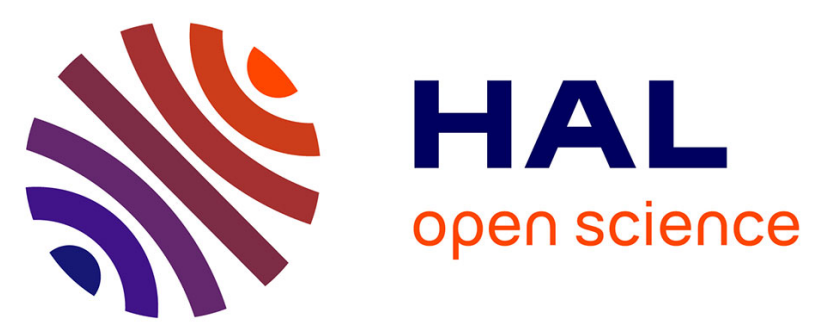

\title{
Analytical Prediction of Magnetic Field in Parallel Double excitation and Spoke-Type Permanent-Magnet Machines Accounting for Tooth-Tips and Shape of Polar Pieces
}

\author{
Kamel Boughrara, Rachid Ibtiouen, Thierry Lubin
}

\section{To cite this version:}

Kamel Boughrara, Rachid Ibtiouen, Thierry Lubin. Analytical Prediction of Magnetic Field in Parallel Double excitation and Spoke-Type Permanent-Magnet Machines Accounting for Tooth-Tips and Shape of Polar Pieces. IEEE Transactions on Magnetics, 2012, 18 p. 10.1109/TMAG.2012.2186587 . hal00673949

\section{HAL Id: hal-00673949 \\ https://hal.science/hal-00673949}

Submitted on 24 Feb 2012

HAL is a multi-disciplinary open access archive for the deposit and dissemination of scientific research documents, whether they are published or not. The documents may come from teaching and research institutions in France or abroad, or from public or private research centers.
L'archive ouverte pluridisciplinaire HAL, est destinée au dépôt et à la diffusion de documents scientifiques de niveau recherche, publiés ou non, émanant des établissements d'enseignement et de recherche français ou étrangers, des laboratoires publics ou privés. 


\title{
Analytical Prediction of Magnetic Field in Parallel Double Excitation and Spoke-Type Permanent-Magnet Machines Accounting for Tooth- Tips and Shape of Polar Pieces
}

\author{
K. Boughrara ${ }^{1}$, R. Ibtiouen ${ }^{2}$ and T. Lubin ${ }^{3}$ \\ ${ }^{1}$ Université de Khemis-Miliana (LESI), Route de Theniet El-had, Khemis-Miliana, 44225, Algeria \\ ${ }^{2}$ Ecole Nationale Polytechnique (LRE-ENP), Algiers, 10, av. Pasteur, El Harrach, BP182, 16200, Algeria \\ ${ }^{3}$ Groupe de Recherche en Electrotechnique et Electronique de Nancy, University Henri Poincaré, 54506 Vandoeuvre-lès-Nancy \\ Cedex, France
}

\begin{abstract}
This paper presents an analytical method based on subdomain method for the computation of open circuit, armature reaction and on-load magnetic field distribution in integer slot winding parallel double excitation and Spoke-Type tangential permanent-magnet machines. The proposed model takes into account for stator and rotor slots tooth tips and shape of polar piece. A 2D exact analytical solution of magnetic field distribution is established. It involves solution of Laplace's and Poisson's equations in semi-closed stator and rotor slots, airgap, buried permanent magnets into rotor semi-closed slots, and non magnetic region under magnets. Obtained exact analytical results of open circuit, armature reaction and on-load magnetic field distribution are verified with those issued from finite element method (FEM).
\end{abstract}

Index Terms - Exact analytical calculation, finite element method, magnetic field distribution, parallel double excitation, SpokeType permanent magnet machines.

\section{INTRODUCTION}

$\mathrm{D}_{\mathrm{b}}$ ouble excitation machines are synchronous machines that have two coexisting excitation field sources: permanent magnets and wound field excitation. The goal behind the use of double excitation principle is to combine advantages of permanent-magnet excited machines and wound field synchronous machines. The combination of permanent magnets and wound field excitation constitutes an additional degree of freedom which can be used to optimize the energy consumption of the electric propulsion system. Among various double excitation machines, parallel double excitation machines have some advantages than series double excitation machines [1]-[5]. The flux created by excitation coils and permanent magnets have different trajectories and the flux created by excitation coils doesn't pass through permanent magnets, hence the demagnetization risk is avoided. Depending on DC excitation current direction, excitation coils can either be used to enhance or decrease excitation flux passing through armature windings.

There are no authors who applied analytical model for predicting magnetic field in this type of hybrid excitation machines with slotted rotor and stator and buried tangential permanent magnet taking into account rotor and stator tooth tips and real structure of polar piece. Analytical methods are useful tools for a first evaluation of electrical motors performances and for design optimization. However, finite element method is used at the final stage of the design. In reference [5], exact analytical calculation of magnetic field distribution is developed for a slotted stator series double excitation machine, where coils current excitation are situated in the slotted rotor with radial surface mounted permanent magnets. There are other structures of double excitation permanent magnet machines with complicated geometry [6][8], where the study is done by finite element method only.
Permanent magnet brushless dc (BLDC) motors have been widely used due to their many advantages such as high torque and high efficiency. In particular, the spoke-type BLDC motor, which can concentrate the flux from low cost ferrite permanent magnets, has a high torque density per unit volume resulting from the additional reluctance torque. The prediction of open circuit, armature reaction and on load magnetic field distribution of this type of motors is done generally by the finite element method (FEM) [9]-[11] and there are very few authors which use analytical methods [12]-[13]. This is due to the structure of those machines, where buried tangential PM region has high height and low width with presence of non magnetic region under magnet. In reference [12], Lin et all. used conformal transformation of rotor and stator slots to determine a relative permeance function to take into account the rotor and stator slotting effects. In reference [13], authors used also a Schwarz-Christoffel transformation to determine d-axial and q-axial airgap magnetic field. Conformal transformation leads to an approximation of magnetic field distribution in a simplified structure of slotted stator spoketype permanent magnet motors. Investigations in applying analytical and numerical conformal mapping have been done in [14] and show the inaccuracy of conformal mapping method in magnetic field modeling of this type of machines. This is not due to the stator slotting effect but to the presence of a deep and small thickness of permanent magnet region. To the author's knowledge, there is no study on the slotted or slotless Spoke-Type PM machines that solves analytically Poisson's and Laplace's equations. However, many authors have used exact analytical method based on subdomain model to take into account stator slotting effect and tooth tips in open circuit and armature reaction magnetic field with circular and tubular radial surface mounted and inset permanent magnet motors [15]-[24]. Inset PM subregion in PM machines is not deep and the width is high. So, it can be modeled accurately 
with conformal mapping [25]. In references [15]-[17], authors have developed an exact analytical solution based on subdomain model for predicting open circuit and armature reaction in slotted stator radial surface mounted permanent magnet motor taking into account tooth tips. In [18] and [19], scalar potential magnetic field is used to predict open circuit magnetic field in slotted stator surface and inset radial permanent magnet motors. Authors in [20] and [21] have developed an exact analytical solution based on subdomain model for predicting open circuit and armature reaction in tubular surface mounted and inset radial permanent magnet machines. In [22]-[24], the authors have developed respectively an exact analytical solution also based on subdomain model for flux switching permanent magnet machines and a tubular slotless stator with axially magnetized permanent magnet in rotor.

In this paper, an exact analytical prediction based on subdomain model of open circuit, armature reaction and onload magnetic field distribution in integer slot winding parallel double excitation and spoke-type tangential permanent magnet machines is presented. It involves the solution of Poisson's and Laplace's equations in semi-closed stator slots, buried permanent magnets placed in semi-closed slots, rotor double excitation semi-closed slots, airgap and non magnetic region under permanent magnets. To handle permanent magnets, rotor and stator slots current at the same time, The magnetic vector potential formulation is used. All results from the developed analytical model are then compared to those found by the finite element method (FEM).

\section{Magnetic Field Solution in PARALlel Double EXCITATION PM MACHINES}

Figs. 1 and 2 show the machine model where region I represents the air gap, region II the magnets, regions III and V the stator semi-closed slots, region IV a non magnetic material under magnets, region VI the rotor slots at the top of permanent magnets, regions VII and VIII the rotor excitation semi-closed slots. The model is formulated in vector potential and two-dimensional polar coordinates with the following assumptions.

The stator and rotor cores are assumed to be infinitely permeable.

Eddy current effects are neglected.

The axial length of the machine is infinite i.e. end effects are neglected.

The current density has only one component along the zaxis.

The stator and rotor slots have radial sides.

The partial differential equations for magnetic fields in a continuous and isotopic region in term of vector potential $A$ which has one component in $z$ direction and independent of $z$ can be expressed by

$$
\begin{aligned}
& \nabla^{2} A=0, \text { in region I, IV, V, VI and VIII } \\
& \nabla^{2} A=-\mu_{0} \nabla \times M, \text { in region II } \\
& \nabla^{2} A=-\mu_{0} J, \text { in region III }
\end{aligned}
$$

$$
\nabla^{2} A=-\mu_{0} J_{f}, \text { in region VII }
$$

where $M$ is the magnetization of permanent magnets, $J$ the stator slots current density, $\mathrm{J}_{\mathrm{f}}$ the excitation rotor slots current density and $\mu_{0}$ the permeability of vacuum.

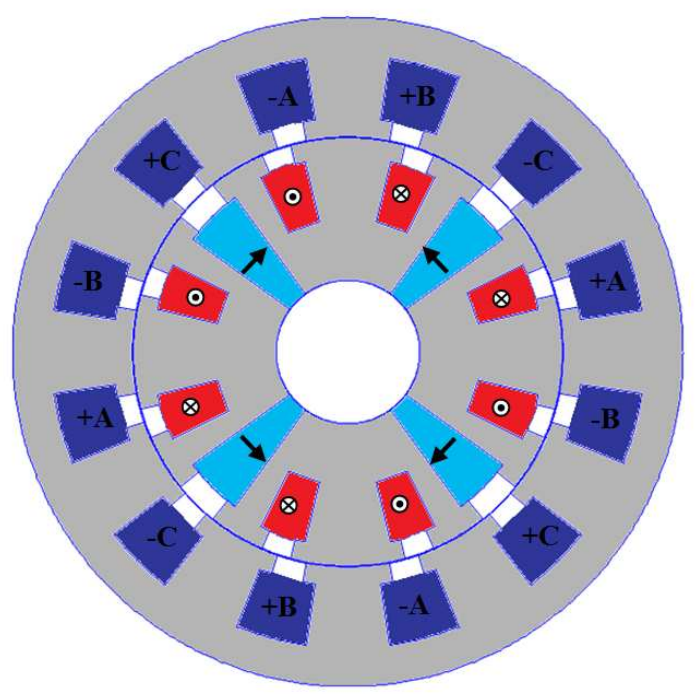

Fig. 1. Studied parallel double excitation PM machine.

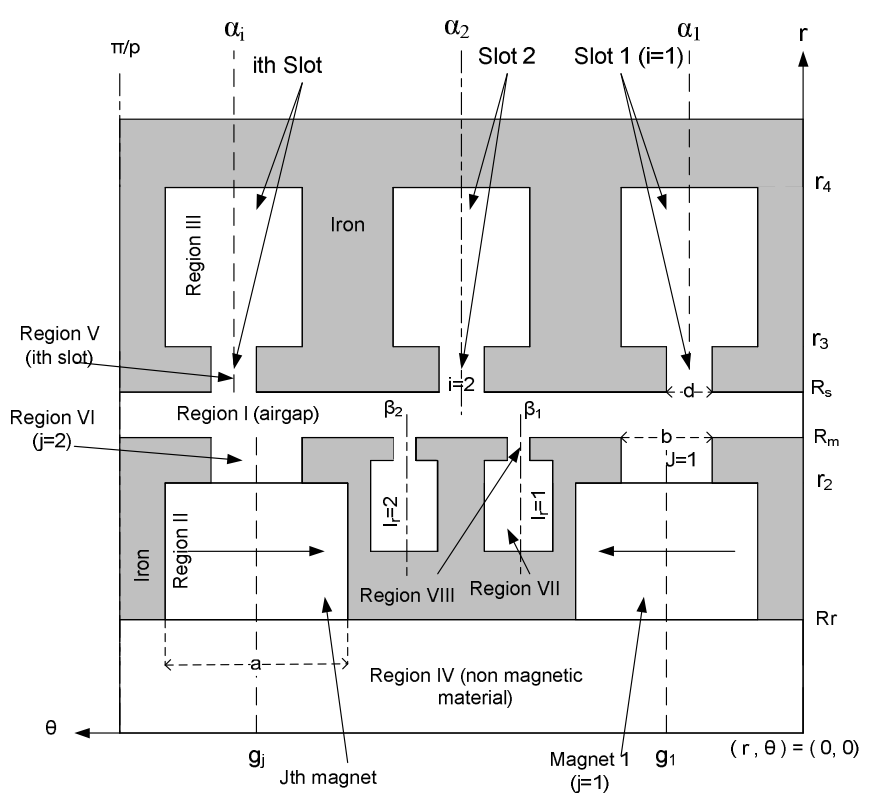

Fig. 2. Simplified model. 


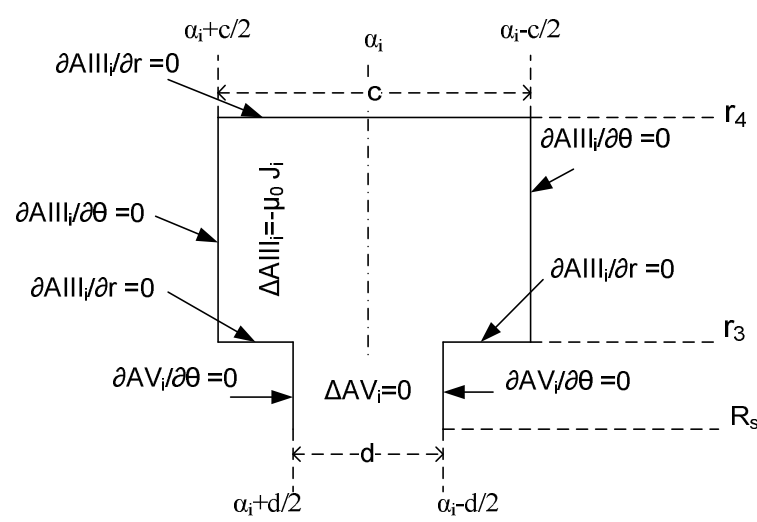

Fig. 3. ith stator semi-closed slot subdomains

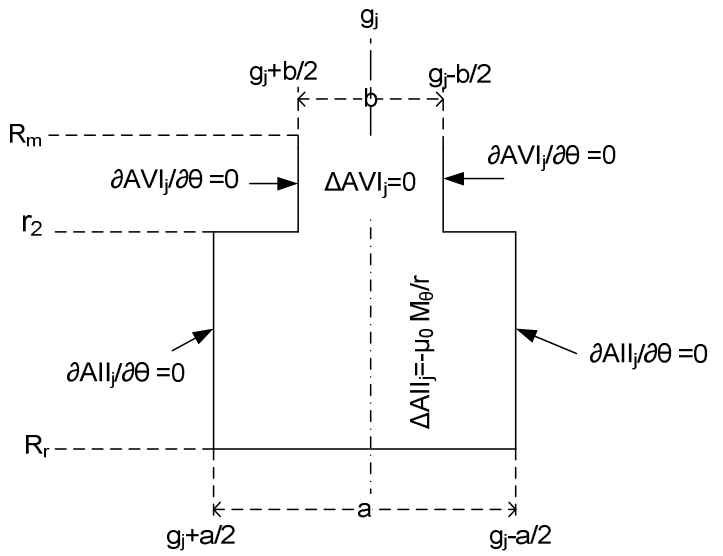

Fig. 4. jth permanent magnet subdomains

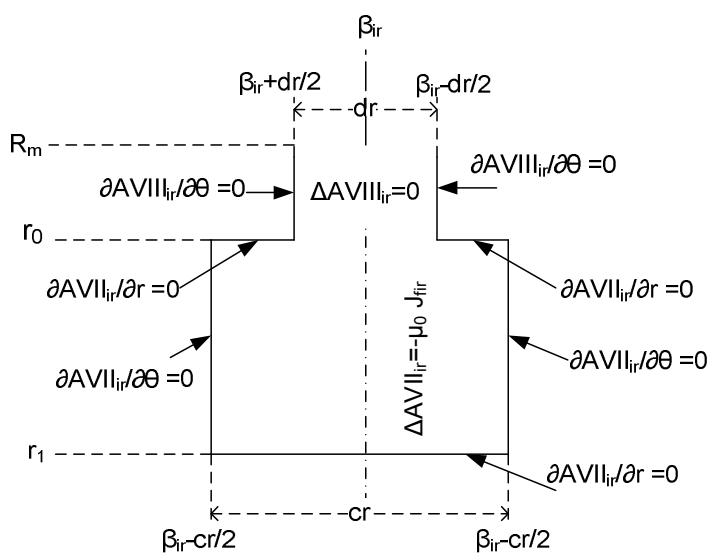

Fig. 5. irth rotor semi-closed slot subdomains

The field vectors $B$ and $H$, in the different regions, are coupled by

$B=\mu_{0} H$, in regions I, III, IV, V, VI, VII and VIII

where $B_{r}=\mu_{0} H_{r}, B_{\theta}=\mu_{0} H_{\theta}$.

$$
B=\mu_{0} \mu_{r} H+\mu_{0} M \text {, in region II }
$$

where $B_{r}=\mu_{0} \mu_{r} H_{r}+\mu_{0} M_{r}, B_{\theta}=\mu_{0} \mu_{r} H_{\theta}+\mu_{0} M_{\theta}$ and $\mu_{r}$ is the relative recoil permeability of permanent magnets. Radial and circumferential flux density components are deduced from $A$ by

$$
B_{r}=\frac{1}{r} \frac{\partial A}{\partial \theta} ; B_{\theta}=-\frac{\partial A}{\partial r}
$$

A. General Solution of Poisson's Equation in Stator Semiclosed Slot Subdomain (Region III)

In each slot subdomain (i) of region III (Fig. 3), we have to solve Poisson's equation

$$
\frac{\partial^{2} A I I I_{i}}{\partial r^{2}}+\frac{1}{r} \frac{\partial A I I I_{i}}{\partial r}+\frac{1}{r^{2}} \frac{\partial^{2} A I I I_{i}}{\partial \theta^{2}}=-\mu_{0} J_{i}
$$

where $J_{i}$ is the current density in the slot $i$.

As shown in Fig. 3, the ith slot subdomain (region III) where $i$ vary from 1 to $Q_{s}$ ( $Q_{s}$ is the number of stator slots) is associated with boundary conditions at the bottom and at each sides of the slot as

$$
\begin{aligned}
& \left.\frac{\partial A I I I_{i}}{\partial \theta}\right|_{\theta=\alpha_{i}-\frac{c}{2}}=0 \text { and }\left.\frac{\partial A I I I_{i}}{\partial \theta}\right|_{\theta=\alpha_{i}+\frac{c}{2}}=0 \\
& \left.\frac{\partial A I I_{i}}{\partial r}\right|_{r=r_{4}}=0
\end{aligned}
$$

where $\alpha_{i}$ is the angular position of the ith slot and $c$ the slot opening in radian.

The boundary condition (9), leads to the eigenvalues and eigenfunctions of the partial differential equation (8) as shown in details in [26]. The eigenfunctions are called spatial frequencies of the considered region [23]. The boundary condition (10), leads to the general solution of equation (8) with only two integration constants $\mathrm{C}_{\mathrm{i}, 0}$ and $\mathrm{C}_{\mathrm{i}, \mathrm{m}}$ as shown in (11).

From above boundary conditions (9) and (10), the solution of (8) using the method of separation of variables is

$$
\begin{aligned}
& A I_{i}(r, \theta)=C_{i, 0}+\frac{1}{2} \mu_{0} J_{i} r_{4}^{2} \ln (r)-\frac{1}{4} \mu_{0} J_{i} r^{2} \\
& +\sum_{m=1}^{\infty} C_{i, m}\left[\left(\frac{r}{r_{4}}\right)^{\frac{m \pi}{c}}-\left(\frac{r}{r_{4}}\right)^{-\frac{m \pi}{c}}\right] \cos \left(\frac{m \pi}{c}\left(\theta-\alpha_{i}+\frac{c}{2}\right)\right)
\end{aligned}
$$

where $\mathrm{m}$ is a positive integer.

B. General Solution of Laplace's Equation in Stator SemiClosed Slot Subdomain (Region V)

In each stator semi-closed slot subdomain (i) of region $\mathrm{V}$ (Figs. 2, 3), we have to solve Laplace's equation

$$
\frac{\partial^{2} A V_{i}}{\partial r^{2}}+\frac{1}{r} \frac{\partial A V_{i}}{\partial r}+\frac{1}{r^{2}} \frac{\partial^{2} A V_{i}}{\partial \theta^{2}}=0
$$

As shown in Fig. 3, the ith slot subdomain (region V) where $i$ vary from 1 to $Q_{s}$ ( $Q_{s}$ number of stator slots) is associated with the following boundary conditions

$$
\left.\frac{\partial A V_{i}}{\partial \theta}\right|_{\theta=\alpha_{i}-\frac{d}{2}}=0 \text { and }\left.\frac{\partial A V_{i}}{\partial \theta}\right|_{\theta=\alpha_{i}+\frac{d}{2}}=0
$$

where $d$ is the semi-closed slot opening in radian.

From above boundary conditions (13), the solution of (12) using the method of separation of variables is 


$$
\begin{aligned}
& A V_{i}(r, \theta)=A 11_{i, 0}+A 12_{i, 0} \ln (r) \\
& +\sum_{k=1}^{\infty}\left(A 11_{i, k} r^{-\frac{k \pi}{d}}+A 12_{i, k} r^{\frac{k \pi}{d}}\right) \cos \left(\frac{k \pi}{d}\left(\theta-\alpha_{i}+\frac{d}{2}\right)\right)
\end{aligned}
$$

where $\mathrm{k}$ is a positive integer.

\section{General Solution of Poisson's Equation in Permanent Magnet Subdomain (Region II)}

In each permanent magnet subdomain (j) of region II (Figs. 2 and 4), we have to solve Poisson's equation (2). The magnetization of parallel double excitation motor is purely tangential. Equation (2) is then reduced to

$$
\frac{\partial^{2} A I I_{j}}{\partial r^{2}}+\frac{1}{r} \frac{\partial A I I_{j}}{\partial r}+\frac{1}{r^{2}} \frac{\partial^{2} A I I_{j}}{\partial \theta^{2}}=-\mu_{0} \frac{M_{\theta}}{r}
$$

where $M_{\theta}=M_{j}=(-1)^{j} \frac{B_{\text {rem }}}{\mu_{0}}$

For a $2 p$ poles machine, $\mathrm{j}$ vary from 1 to $2 p$ and $B_{r e m}$ is the remanence of magnetization.

As shown in Fig. 4, the jth magnet subdomain (region II) is associated with the following boundary conditions

$$
\left.\frac{\partial A I I_{j}}{\partial \theta}\right|_{\theta=g_{j}-\frac{a}{2}}=0 \text { and }\left.\frac{\partial A I I_{j}}{\partial \theta}\right|_{\theta=g_{j}+\frac{a}{2}}=0
$$

where $g_{j}$ is the angular position of the $j$ th magnet and $a$ the magnet opening in radian.

From above boundary conditions (16), the general solution of (15) using the method of separation of variables is given by

$$
\begin{gathered}
A I I_{j}(r, \theta)=A 5_{j, 0}+A 6_{j, 0} \ln (r)-\mu_{0} M_{j} r \\
+\sum_{m=1}^{\infty}\left(A 5_{j, m} r^{-\frac{m \pi}{a}}+A 6_{j, m} r^{\frac{m \pi}{a}}\right) \cos \left(\frac{m \pi}{a}\left(\theta-g_{j}+\frac{a}{2}\right)\right)
\end{gathered}
$$

\section{General Solution of Laplace's Equation in Rotor Semi-} Closed Slot Subdomain (Region VI)

In each rotor semi-closed slot subdomain (j) of region VI (Figs. 2, 4), we have to solve Laplace's equation

$$
\frac{\partial^{2} A V I_{j}}{\partial r^{2}}+\frac{1}{r} \frac{\partial A V I_{j}}{\partial r}+\frac{1}{r^{2}} \frac{\partial^{2} A V I_{j}}{\partial \theta^{2}}=0
$$

As shown in Fig. 4, the jth slot subdomain (region VI) where $\mathrm{j}$ vary from 1 to $2 p$ is associated with the following boundary conditions

$$
\left.\frac{\partial A V I_{j}}{\partial \theta}\right|_{\theta=g_{j}-\frac{b}{2}}=0 \text { and }\left.\frac{\partial A V I_{j}}{\partial \theta}\right|_{\theta=g_{j}+\frac{b}{2}}=0
$$

where $b$ is the semi-closed slot opening in radian.

From above boundary conditions (19), the solution of (18) using the method of separation of variables is

$$
\begin{aligned}
& A V I_{j}(r, \theta)=A 13_{j, 0}+A 14_{j, 0} \ln (r) \\
& +\sum_{k=1}^{\infty}\left(A 13_{j, k} r^{-\frac{k \pi}{b}}+A 14_{j, k} r^{\frac{k \pi}{b}}\right) \cos \left(\frac{k \pi}{b}\left(\theta-g_{j}+\frac{b}{2}\right)\right)
\end{aligned}
$$

\section{E. General Solution of Laplace's Equation in Airgap Subdomain (Region I)}

The Laplace equation (1) in the airgap subdomain (region I) which is an annular domain delimited by the radii $R_{m}$ and $R_{s}$ (Fig. 2) is given by

$$
\frac{\partial^{2} A I}{\partial r^{2}}+\frac{1}{r} \frac{\partial A I}{\partial r}+\frac{1}{r^{2}} \frac{\partial^{2} A I}{\partial \theta^{2}}=0
$$

Taking into account the periodicity boundary condition between $\theta=0$ and $\theta=2 \pi / p$ for the studied machine with integer slot winding, the solution of equation (21) is

$$
\begin{aligned}
A I(r, \theta)= & \sum_{n=1}^{\infty}\left(A 1_{n} r^{n p}+A 2_{n} r^{-n p}\right) \sin (n p \theta) \\
& +\left(A 3_{n} r^{n p}+A 4_{n} r^{-n p}\right) \cos (n p \theta)
\end{aligned}
$$

where $\mathrm{n}$ is a positive integer.

\section{F. General Solution of Laplace's Equation in the Non Magnetic Subdomain (Region IV)}

The Laplace's equation (1) in the non magnetic subdomain region IV which is an annular domain delimited by the radii $R_{i}$ and $R_{r}$ and where the relative recoil permeability is equal to 1 is given by

$$
\frac{\partial^{2} A I V}{\partial r^{2}}+\frac{1}{r} \frac{\partial A I V}{\partial r}+\frac{1}{r^{2}} \frac{\partial^{2} A I V}{\partial \theta^{2}}=0
$$

Taking into account the periodicity boundary condition between $\theta=0$ and $\theta=2 \pi / p$, the general solution of (23) is

$$
\begin{aligned}
A I V(r, \theta)= & \sum_{n=1}^{\infty}\left(A 7_{n} r^{n p}+A 8_{n} r^{-n p}\right) \sin (n p \theta) \\
& +\left(A 9_{n} r^{n p}+A 10_{n} r^{-n p}\right) \cos (n p \theta)
\end{aligned}
$$

The magnetic vector potential must be finite in region IV. In this case, constants $\mathrm{A} 8_{\mathrm{n}}$ and $\mathrm{A} 10_{\mathrm{n}}$ are equals to zero and equation (24) is reduced to

$$
\begin{array}{r}
A I V(r, \theta)=\sum_{n=1}^{\infty} A 7_{n} r^{n p} \sin (n p \theta) \\
+A 9_{n} r^{n p} \cos (n p \theta)
\end{array}
$$

\section{G. General Solution of Poisson's Equation in Rotor} Excitation Coil Slot Subdomain (Region VII)

In each rotor slot subdomain (ir) of region VII, we have to solve Poisson's equation (26)

$\frac{\partial^{2} A V I I_{i r}}{\partial r^{2}}+\frac{1}{r} \frac{\partial A V I I_{i r}}{\partial r}+\frac{1}{r^{2}} \frac{\partial^{2} A V I I_{i r}}{\partial \theta^{2}}=-\mu_{0} J_{f_{i r}}$

where $J_{f i r}$ is the current density in rotor slot $i r$.

As shown in Fig. 5, the irth slot subdomain (region VII) where ir vary from 1 to $N_{r}\left(N_{r}\right.$ is total number of rotor excitation slots) is associated with the following boundary conditions 


$$
\begin{aligned}
& \left.\frac{\partial A V I I_{i r}}{\partial \theta}\right|_{\theta=\beta_{i r}-\frac{c r}{2}}=0 \text { and }\left.\frac{\partial A V I I_{i r}}{\partial \theta}\right|_{\theta=\beta_{i r}+\frac{c r}{2}}=0 \\
& \left.\frac{\partial A V I I_{i r}}{\partial r}\right|_{r=r_{1}}=0
\end{aligned}
$$

where $\beta_{i r}$ is the angular position of the irth slot and $c_{r}$ the rotor slot opening in radian.

From the above boundary conditions (27) and (28), the solution of (26) using the method of separation of variables is

$$
\begin{gathered}
A V I I_{i r}(r, \theta)=C 1_{i r, 0}+\frac{1}{2} \mu_{0} J_{f_{i r}} r_{1}^{2} \ln (r)-\frac{1}{4} \mu_{0} J_{f_{i r}} r^{2} \\
+\sum_{m=1}^{\infty} C 1_{i r, m}\left[\left(\frac{r}{r_{1}}\right)^{\frac{m \pi}{c_{r}}}-\left(\frac{r}{r_{1}}\right)^{-\frac{m \pi}{c_{r}}}\right] \cdot \quad \text { (29) } \\
\cos \left(\frac{m \pi}{c_{r}}\left(\theta-\beta_{i r}+\frac{c_{r}}{2}\right)\right)
\end{gathered}
$$

\section{H. General Solution of Laplace's Equation in Rotor} Excitation Coil Slot Subdomain (Region VIII)

In each semi-closed slot subdomain (ir) of region VIII (Fig. 5), we have to solve Laplace's equation

$$
\frac{\partial^{2} A V I I I_{i r}}{\partial r^{2}}+\frac{1}{r} \frac{\partial A V I I I_{i r}}{\partial r}+\frac{1}{r^{2}} \frac{\partial^{2} A V I I I_{i r}}{\partial \theta^{2}}=0
$$

As shown in Fig. 5, the irth slot subdomain (region VIII) where ir vary from 1 to $N_{r}$ is associated with the following boundary conditions

$$
\left.\frac{\partial A V I I I_{i r}}{\partial \theta}\right|_{\theta=\beta_{i r}-\frac{d r}{2}}=0 \text { and }\left.\frac{\partial A V I I I_{i r}}{\partial \theta}\right|_{\theta=\beta_{i r}+\frac{d r}{2}}=0
$$

where $d r$ is the semi-closed slot opening in radian.

From previous boundary conditions (31), the solution of partial differential equation (30) using the method of separation of variables is

$$
\begin{aligned}
A_{V I I I}(r, \theta)= & A 15_{i r, 0}+A 16_{i r, 0} \ln (r) \\
+ & \sum_{k=1}^{\infty}\left(A 15_{i r, k} r^{-\frac{k \pi}{d r}}+A 16_{i r, k} r^{\frac{k \pi}{d r}}\right) . \\
& \cos \left(\frac{k \pi}{d r}\left(\theta-\beta_{i r}+\frac{d r}{2}\right)\right)
\end{aligned}
$$

\section{BOUNDARY AND INTERFACE CONDITIONS}

To determine Fourier series unknown constants $\mathrm{A} 1_{\mathrm{n}}, \mathrm{A} 2_{\mathrm{n}}$, $\mathrm{A} 3_{\mathrm{n}}, \mathrm{A} 4_{\mathrm{n}}, \mathrm{A} 5_{\mathrm{j}, 0}, \mathrm{~A} 6_{\mathrm{j}, 0}, \mathrm{~A} 5_{\mathrm{j}, \mathrm{m}}, \mathrm{A} 6_{\mathrm{j}, \mathrm{m}}, \mathrm{A} 7_{\mathrm{n}}, \mathrm{A} 9_{\mathrm{n}}, \mathrm{A} 11_{\mathrm{i}, 0}, \mathrm{~A} 12_{\mathrm{i}, 0}$, $\mathrm{A} 11_{\mathrm{i}, \mathrm{k}}, \mathrm{A} 12_{\mathrm{i}, \mathrm{k}}, \mathrm{A} 13_{\mathrm{j}, 0}, \mathrm{~A} 14_{\mathrm{j}, 0}, \mathrm{~A} 13_{\mathrm{j}, \mathrm{k}}, \mathrm{A} 14_{\mathrm{j}, \mathrm{k}, \mathrm{C}} \mathrm{C}_{\mathrm{i}, 0}, \mathrm{C}_{\mathrm{i}, \mathrm{m}}, \mathrm{C} 1_{\mathrm{ir}, 0}$, $\mathrm{C} 1_{\mathrm{ir}, \mathrm{m}}, \mathrm{A} 15_{\mathrm{ir}, 0}, \mathrm{~A} 16_{\mathrm{ir}, 0}, \mathrm{~A} 15_{\mathrm{ir}, \mathrm{k}}$ and $\mathrm{A} 16_{\mathrm{ir}, \mathrm{k}}$, boundary and interface conditions should be introduced.

The interface conditions between regions IV and II at $R_{r}$ are

$$
A I I_{j}\left(R_{r}, \theta\right)=A I V\left(R_{r}, \theta\right)
$$

where $g_{j}-\frac{a}{2} \leq \theta \leq g_{j}+\frac{a}{2}$.

$$
H I I_{\theta_{j}}\left(R_{r}, \theta\right)=H I V_{\theta}\left(R_{r}, \theta\right)
$$

where $g_{j}-\frac{a}{2} \leq \theta \leq g_{j}+\frac{a}{2}$.

$H_{I V} V_{\theta}\left(R_{r}, \theta\right)=0$ elsewhere.

The interface conditions between regions II and VI at $r_{2}$ are

$$
A V I_{j}\left(r_{2}, \theta\right)=A I I_{j}\left(r_{2}, \theta\right)
$$

where $g_{j}-\frac{b}{2} \leq \theta \leq g_{j}+\frac{b}{2}$.

$$
H V I_{\theta_{j}}\left(r_{2}, \theta\right)=H I I_{\theta_{j}}\left(r_{2}, \theta\right)
$$

where $g_{j}-\frac{b}{2} \leq \theta \leq g_{j}+\frac{b}{2}$.

$H I I_{\theta_{j}}\left(r_{2}, \theta\right)=0$ elsewhere.

The interface condition between regions I and VI at $\mathrm{R}_{\mathrm{m}}$ is

$$
A V I_{j}\left(R_{m}, \theta\right)=A I\left(R_{m}, \theta\right)
$$

where $g_{j}-\frac{b}{2} \leq \theta \leq g_{j}+\frac{b}{2}$.

The interface condition between regions I and VIII at $\mathrm{R}_{\mathrm{m}}$ is

$$
A I\left(R_{m}, \theta\right)=A \operatorname{VIII}_{i r}\left(R_{m}, \theta\right)
$$

where $\beta_{i r}-\frac{d_{r}}{2} \leq \theta \leq \beta_{i r}+\frac{d_{r}}{2}$.

The interface conditions between regions I, VI and VIII at $\mathrm{R}_{\mathrm{m}}$ are

$$
H I_{\theta}\left(R_{m}, \theta\right)=H V I_{\theta_{j}}\left(R_{m}, \theta\right)
$$

for $g_{j}-\frac{b}{2} \leq \theta \leq g_{j}+\frac{b}{2}$ and

$$
H I_{\theta}\left(R_{m}, \theta\right)=H V I I I_{\theta_{i r}}\left(R_{m}, \theta\right)
$$

for $\beta_{i r}-\frac{d_{r}}{2} \leq \theta \leq \beta_{i r}+\frac{d_{r}}{2}$ and

$H I_{\theta}\left(R_{m}, \theta\right)=0$ elsewhere.

The interface conditions between regions $\mathrm{I}$ and $\mathrm{V}$ at $R_{s}$ are

$$
A I\left(R_{s}, \theta\right)=A V_{i}\left(R_{s}, \theta\right)
$$

where $\alpha_{i}-\frac{d}{2} \leq \theta \leq \alpha_{i}+\frac{d}{2}$.

$$
H I_{\theta}\left(R_{s}, \theta\right)=H V_{\theta_{i}}\left(R_{s}, \theta\right)
$$

where $\alpha_{i}-\frac{d}{2} \leq \theta \leq \alpha_{i}+\frac{d}{2}$.

$H I_{\theta}\left(R_{s}, \theta\right)=0$ elsewhere.

The interface conditions between regions III and $\mathrm{V}$ at $r_{3}$ are

$$
A I I I_{i}\left(r_{3}, \theta\right)=A V_{i}\left(r_{3}, \theta\right)
$$


where $\alpha_{i}-\frac{d}{2} \leq \theta \leq \alpha_{i}+\frac{d}{2}$.

$$
H I I I_{\theta_{i}}\left(r_{3}, \theta\right)=H V_{\theta_{i}}\left(r_{3}, \theta\right)
$$

where $\alpha_{i}-\frac{d}{2} \leq \theta \leq \alpha_{i}+\frac{d}{2}$.

$$
H_{I I I}\left(r_{3}, \theta\right)=0 \text { elsewhere. }
$$

The interface conditions between regions VII and VIII at $r_{0}$ are

$$
\begin{aligned}
& A V I I_{i r}\left(r_{0}, \theta\right)=A V I I I_{i r}\left(r_{0}, \theta\right) \\
& \text { where } \beta_{i r}-\frac{d_{r}}{2} \leq \theta \leq \beta_{i r}+\frac{d_{r}}{2} \\
& H_{V I I} I_{\theta i r}\left(r_{0}, \theta\right)=H V I I I_{\theta i r}\left(r_{0}, \theta\right) \\
& \text { where } \beta_{i r}-\frac{d_{r}}{2} \leq \theta \leq \beta_{i r}+\frac{d_{r}}{2} \\
& H V I I_{\theta i r}\left(r_{0}, \theta\right)=0 \text { elsewhere }
\end{aligned}
$$

Interface conditions (33) to (45) concern regions with different subdomain frequencies which need Fourier series expansions to satisfy equalities of vector potential and magnetic field at each interface radius.

Interface condition (33) represents also the continuity of radial flux density at the permanent magnet opening at $R_{r}$. According to Fourier series expansion, we obtain two equations as

$$
\begin{aligned}
A 5_{j, 0}+A 6_{j, 0} \ln \left(R_{r}\right)-M_{j} \mu_{0} R_{r} \\
=\frac{1}{a} \int_{g_{j}-\frac{a}{2}}^{g_{j}+\frac{a}{2}} A I V\left(R_{r}, \theta\right) d \theta \\
A 5_{j, m} R_{r}^{-\left(\frac{m \pi}{a}\right)}+A 6_{j, m} R_{r}^{\left(\frac{m \pi}{a}\right)} \\
=\frac{2}{a} \int_{g_{j}-\frac{a}{2}}^{g_{j}+\frac{a}{2}} A I V\left(R_{r}, \theta\right) \cos \left(\frac{m \pi}{a}\left(\theta-g_{j}+\frac{a}{2}\right)\right) d \theta
\end{aligned}
$$

Interface condition (34) gives

$$
\begin{aligned}
& \left(\frac{n p}{\mu_{0}}\right)\left(-A 7_{n} R_{r}^{n p-1}\right) \\
& =\frac{1}{\pi} \sum_{j=1}^{2 p} \int_{g_{j}-\frac{a}{2}}^{g_{j}+\frac{a}{2}} H I I_{\theta j}\left(R_{r}, \theta\right) \sin (n p \theta) d \theta \\
& \left(\frac{n p}{\mu_{0}}\right)\left(-A 9_{n} R_{r}^{n p-1}\right)
\end{aligned}
$$

$$
=\frac{1}{\pi} \sum_{j=1}^{2 p} \int_{g_{j}-\frac{a}{2}}^{g_{j}+\frac{a}{2}} H I I_{\theta_{j}}\left(R_{r}, \theta\right) \cos (n p \theta) d \theta
$$

Fourier series expansion of interface condition (35) between region II and VI at radius $\mathrm{r}_{2}$ gives

$$
\begin{aligned}
& A 13_{j, 0}+A 14_{j, 0} \ln \left(r_{2}\right) \\
= & \frac{1}{b} \int_{g_{j}-\frac{b}{2}}^{g_{j}+\frac{b}{2}} A I I_{j}\left(r_{2}, \theta\right) d \theta \\
& A 13_{j, k} r_{2}^{-\frac{k \pi}{b}}+A 14_{j, k} r_{2}^{\frac{k \pi}{b}} \\
= & \frac{2}{b} \int_{g_{j}-\frac{b}{2}}^{g_{j}+\frac{b}{2}} A I I_{j}\left(r_{2}, \theta\right) \cos \left(\frac{k \pi}{b}\left(\theta-g_{j}+\frac{b}{2}\right)\right) d \theta
\end{aligned}
$$

From interface condition (36), we get

$$
\begin{aligned}
& -\frac{A 6_{j, 0}}{r_{2}} \frac{1}{\mu_{0} \mu_{r}}=\frac{1}{a} \int_{g_{j}-\frac{b}{2}}^{g_{j}+\frac{b}{2}} H V I_{\theta j}\left(r_{2}, \theta\right) d \theta \\
& \quad \frac{1}{\mu_{0} \mu_{r}} \frac{m \pi}{a}\left(A 5_{j, m} r_{2}^{-\frac{m \pi}{a}-1}-A 6_{j, m} r_{2}^{\frac{m \pi}{a}-1}\right) \\
& =\frac{2}{a} \int_{g_{j}-\frac{b}{2}}^{g_{j}+\frac{b}{2}} H V I_{\theta j}\left(r_{2}, \theta\right) \cos \left(\frac{m \pi}{a}\left(\theta-g_{j}+\frac{a}{2}\right)\right) d \theta
\end{aligned}
$$

At radius $\mathrm{R}_{\mathrm{m}}$, Fourier series expansions of the three interface conditions (37) to (39) between regions I, VI and VIII result in 6 equations. Interface condition (37) gives

$$
\begin{aligned}
& A 13_{j, 0}+A 14_{j, 0} \ln \left(R_{m}\right)=\frac{1}{b} \int_{g_{j}-\frac{b}{2}}^{g_{j}+\frac{b}{2}} A I\left(R_{m}, \theta\right) d \theta \\
& A 13_{j, k} R_{m}^{-\frac{k \pi}{b}}+A 14_{j, k} R_{m}^{\frac{k \pi}{b}} \\
& =\frac{2}{b} \int_{g_{j}-\frac{b}{2}}^{g_{j}+\frac{b}{2}} A I\left(R_{m}, \theta\right) \cos \left(\frac{k \pi}{b}\left(\theta-g_{j}+\frac{b}{2}\right)\right) d \theta
\end{aligned}
$$

Fourier series expansion of interface condition (38) gives

$$
\begin{aligned}
& A 15_{i r, 0}+A 16_{i r, 0} \ln \left(R_{m}\right)=\frac{1}{b} \int_{\beta_{i r}-\frac{d r}{2}}^{\beta_{i r}+\frac{d r}{2}} A I\left(R_{m}, \theta\right) d \theta \\
& A 15_{i r, k} R_{m}^{-\frac{k \pi}{d r}}+A 16_{i r, k} R_{m}^{\frac{k \pi}{d r}}
\end{aligned}
$$




$$
=\frac{2}{d r} \int_{\beta_{i r}-\frac{d r}{2}}^{\beta_{i r}+\frac{d r}{2}} A I\left(R_{m}, \theta\right) \cos \left(\frac{k \pi}{d r}\left(\theta-\beta_{i r}+\frac{d r}{2}\right)\right)
$$

Fourier series expansion of interface condition (39) gives

$\frac{n p}{\mu_{0}}\left(-A 1_{n} R_{m}^{n p-1}+A 2_{n} R_{m}^{-n p-1}\right)$

$=\frac{1}{\pi} \sum_{j=1}^{2 p} \int_{g_{j}-\frac{b}{2}}^{g_{j}+\frac{b}{2}} H V I_{\theta_{j}}\left(R_{m}, \theta\right) \sin (n p \theta) d \theta$

$+\frac{1}{\pi} \sum_{i r=1}^{N_{r}} \int_{\beta_{i r}-\frac{d r}{2}}^{\beta_{i r}+\frac{d r}{2}} H V I I I_{\theta i r}\left(R_{m}, \theta\right) \sin (n p \theta) d \theta$

$\frac{n p}{\mu_{0}}\left(-A 3_{n} R_{m}^{n p-1}+A 4_{n} R_{m}^{-n p-1}\right)$

$=\frac{1}{\pi} \sum_{j=1}^{2 p} \int_{g_{j}-\frac{b}{2}}^{g_{j}+\frac{b}{2}} H V I_{\theta_{j}}\left(R_{m}, \theta\right) \cos (n p \theta) d \theta$

$+\frac{1}{\pi} \sum_{i r=1}^{N_{r}} \int_{\beta_{i r}-\frac{d r}{2}}^{\beta_{i r}} \int_{\frac{d r}{2}}^{2} H V I I I_{\theta i r}\left(R_{m}, \theta\right) \cos (n p \theta) d \theta$

At radius $\mathrm{R}_{\mathrm{s}}$, Fourier series expansions of interface condition (40) gives

$$
\begin{aligned}
& A 11_{i, 0}+A 12_{i, 0} \ln \left(R_{s}\right)=\frac{1}{d} \int_{\alpha_{i}-\frac{d}{2}}^{\alpha_{i}+\frac{d}{2}} A I\left(R_{s}, \theta\right) d \theta \\
& A 11_{i, k} R_{s}^{-\frac{k \pi}{d}}+A 12_{i, k} R_{s}^{\frac{k \pi}{d}} \\
& =\frac{2}{d} \int_{\alpha_{i}-\frac{d}{2}}^{\alpha_{i}+\frac{d}{2}} A I\left(R_{s}, \theta\right) \cos \left(\frac{k \pi}{d}\left(\theta-\alpha_{i}+\frac{d}{2}\right)\right) d \theta
\end{aligned}
$$

Interface condition (41) gives

$$
\begin{aligned}
& \frac{n p}{\mu_{0}}\left(-A 1_{n} R_{s}^{n p-1}+A 2_{n} R_{s}^{-n p-1}\right) \\
& =\frac{1}{\pi} \sum_{i=1}^{Q_{s}} \int_{\alpha_{i}-\frac{d}{2}}^{\alpha_{i}+\frac{d}{2}} H V_{\theta i}\left(R_{s}, \theta\right) \sin (n p \theta) d \theta \\
& \frac{n p}{\mu_{0}}\left(-A 3_{n} R_{s}^{n p-1}+A 4_{n} R_{s}^{-n p-1}\right)
\end{aligned}
$$

$$
=\frac{1}{\pi} \sum_{i=1}^{Q_{s}} \int_{\alpha_{i}-\frac{d}{2}}^{\alpha_{i}+\frac{d}{2}} H V_{\theta i}\left(R_{s}, \theta\right) \cos (n p \theta) d \theta
$$

Fourier series expansion of interface condition (42) gives

$$
\begin{aligned}
& A 11_{i, 0}+A 12_{i, 0} \ln \left(r_{3}\right)=\frac{1}{d} \int_{\alpha_{i}-\frac{d}{2}}^{\alpha_{i}+\frac{d}{2}} A I I I_{i}\left(r_{3}, \theta\right) d \theta \\
& A 11_{i, k} r_{3}^{-\frac{k \pi}{d}}+A 12_{i, k} r_{3}^{\frac{k \pi}{d}} \\
& =\frac{2}{d} \int_{\alpha_{i}-\frac{d}{2}}^{\alpha_{i}+\frac{d}{2}} A I I I_{i}\left(r_{3}, \theta\right) \cos \left(\frac{k \pi}{d}\left(\theta-\alpha_{i}+\frac{d}{2}\right)\right) d \theta
\end{aligned}
$$

Fourier series expansion of interface condition (43) gives

$$
\begin{aligned}
& \frac{1}{\mu_{0}}\left(-\frac{1}{2} \frac{\mu_{0} J_{i} r_{4}^{2}}{r_{3}}+\frac{1}{2} \mu_{0} J_{i} r_{3}\right) \\
= & \frac{1}{c} \int_{\alpha_{i}-\frac{d}{2}}^{\alpha_{i}+\frac{d}{2}} H V_{\theta i}\left(r_{3}, \theta\right) d \theta \\
- & \frac{C_{i, m}}{\mu_{0}}\left(\frac{m \pi}{c r_{3}}\right)\left(\left(\frac{r_{3}}{r_{4}}\right)^{\frac{m \pi}{c}}+\left(\frac{r_{3}}{r_{4}}\right)^{-\frac{m \pi}{c}}\right) \\
= & \frac{2}{c} \int_{\alpha_{i}-\frac{d}{2}}^{\alpha_{i}+\frac{d}{2}} H V_{\theta i}\left(r_{3}, \theta\right) \cos \left(\frac{m \pi}{c}\left(\theta-\alpha_{i}+\frac{c}{2}\right)\right) d \theta
\end{aligned}
$$

Fourier series expansion of interface condition (44) gives

$$
\begin{aligned}
& A 15_{i r, 0}+A 16_{i r, 0} \ln \left(r_{0}\right) \\
= & \frac{1}{d r} \int_{\beta_{i r}}^{\beta_{i r}+\frac{d r}{2}} A V I I_{i r}\left(r_{0}, \theta\right) d \theta \\
& A 15_{i r, k} r_{0}^{-\frac{k \pi}{d r}}+A 16_{i r, k} r_{0}^{\frac{k \pi}{d r}} \\
= & \frac{2}{d r} \int_{\beta_{i r}}^{\beta_{i r}+\frac{d r}{2}} A V I I_{i r}\left(r_{0}, \theta\right) \cos \left(\frac{k \pi}{d r}\left(\theta-\beta_{i r}+\frac{d r}{2}\right)\right) d \theta
\end{aligned}
$$

Fourier series expansion of interface condition (45) gives

$$
\frac{1}{\mu_{0}}\left(-\frac{1}{2} \frac{\mu_{0} J_{f i r} r_{1}^{2}}{r_{0}}+\frac{1}{2} \mu_{0} J_{f i r} r_{0}\right)
$$




$$
\begin{aligned}
& =\frac{1}{c r} \int_{\beta_{i r}-\frac{d r}{2}}^{\beta_{i r}+\frac{d r}{2}} H V I I I_{\theta i r}\left(r_{0}, \theta\right) d \theta \\
& -\frac{C 1_{i r, m}}{\mu_{0}} \frac{m \pi}{c r r_{0}}\left(\left(\frac{r_{0}}{r_{1}}\right)^{\frac{m \pi}{c r}}+\left(\frac{r_{0}}{r_{1}}\right)^{-\frac{m \pi}{c r}}\right)= \\
& \frac{2}{c r} \int_{\beta_{i r}-\frac{d r}{2}}^{\beta_{i r}+\frac{d r}{2}} H V I I I_{\theta i r}\left(r_{0}, \theta\right) \cos \left(\frac{m \pi}{c r}\left(\theta-\beta_{i r}+\frac{c r}{2}\right)\right) d \theta
\end{aligned}
$$

Some developments of equations (46) to (71) are given in appendix.

From equations (46)-(71) we can calculate the 26 coefficients $\mathrm{A} 1_{\mathrm{n}}, \mathrm{A} 2_{\mathrm{n}}, \mathrm{A} 3_{\mathrm{n}}, \mathrm{A} 4_{\mathrm{n}}, \mathrm{A} 5_{\mathrm{j}, 0}, \mathrm{~A} 6_{\mathrm{j}, 0}, \mathrm{~A} 5_{\mathrm{j}, \mathrm{m}}, \mathrm{A} 6_{\mathrm{j}, \mathrm{m}}, \mathrm{A} 7_{\mathrm{n}}$, $\mathrm{A} 9_{\mathrm{n}}, \mathrm{A} 11_{\mathrm{i}, 0}, \mathrm{~A} 12_{\mathrm{i}, 0}, \mathrm{~A} 11_{\mathrm{i}, \mathrm{k}}, \mathrm{A} 12_{\mathrm{i}, \mathrm{k}}, \mathrm{A} 13_{\mathrm{j}, 0}, \mathrm{~A} 14_{\mathrm{j}, 0}, \mathrm{~A} 13_{\mathrm{j}, \mathrm{k}}, \mathrm{A} 14_{\mathrm{j}, \mathrm{k}}$ $\mathrm{C}_{\mathrm{i}, 0}, \mathrm{C}_{\mathrm{i}, \mathrm{m}}, \mathrm{C} 1_{\mathrm{ir}, 0}, \mathrm{C} 1_{\mathrm{ir}, \mathrm{m}}, \mathrm{A} 15_{\mathrm{ir}, 0}, \mathrm{~A} 16_{\mathrm{ir}, 0}, \mathrm{~A} 15_{\mathrm{ir}, \mathrm{k}}, \mathrm{A} 16_{\mathrm{ir}, \mathrm{k}}$ with a given number of harmonics for $\mathrm{n}, \mathrm{m}$ and $\mathrm{k}$.

Spoke-Type PM machine shown in Fig. 6 is a particular case of parallel double excitation PM machine. The analytical developments are the same where subregions VII and VIII are omitted.

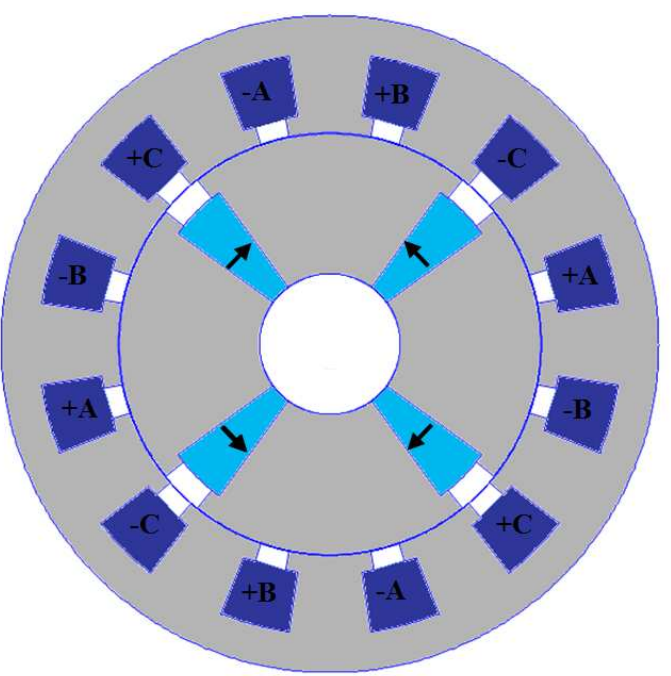

Fig. 6. Studied Spoke-Type PM machine.

\section{RESULTS AND VALIDATION}

The developed exact analytical method based on subdomain model for parallel double excitation and SpokeType permanent magnet machines taking into account rotor and stator slot tooth tips and real structure of polar piece is used to determine open circuit, armature reaction and on load magnetic field distribution in the middle of the airgap. The main dimensions and parameters of the studied machine are given in Table I. Then, analytical results are verified by finite element method (FEM) [27]. For the FE simulations, we have used 92882 nodes and 184906 elements.

In order to calculate armature reaction, on load and rotor double excitation magnetic field distribution, stator current density in slots is defined by a matrix connection between phase current and stator slots as

$$
C=\left[\begin{array}{cccccccccccc}
1 & 0 & 0 & -1 & 0 & 0 & 1 & 0 & 0 & -1 & 0 & 0 \\
0 & 0 & 1 & 0 & 0 & -1 & 0 & 0 & 1 & 0 & 0 & -1 \\
0 & -1 & 0 & 0 & 1 & 0 & 0 & -1 & 0 & 0 & 1 & 0
\end{array}\right]
$$

The studied machine is fed by $120^{\circ}$ rectangular currents and has an integer number of slot per pole and per phase (Table I). Stator current densities in slots are defined as

$$
J_{i}=\frac{N_{c}}{S} C^{T}\left[\begin{array}{lll}
I_{m} & 0 & -I_{m}
\end{array}\right]
$$

where $S$ is the area of slot, $N_{c}$ the number of conductors in slot and $I_{m}$ the stator phase peak current.

Rotor double excitation current density for one pole pair is defined as

$$
J_{f_{n r}}=\frac{N_{f} I_{f}}{S_{f}}\left[\begin{array}{llll}
-1 & -1 & 1 & 1
\end{array}\right]
$$

where $N_{f}$ is the number of conductors in slot, $I_{f}$ the DC excitation current and $S_{f}$ the surface of rotor slot.

Analytical calculation of magnetic field can be done for one pole pair to minimize the number of harmonics for $\mathrm{n}, \mathrm{k}$ and $\mathrm{m}$ and to reduce the computation time. It should be noted that the number of harmonics are defined carefully and including proper scaling of machine model dimensions to get good results and avoid solution to diverge [22]. Finite element method which uses a meshed geometry has a limited accuracy related to the density of the mesh. Analytical subdomain model based on Fourier theory exhibits a similar problem. The inaccuracies of the proposed method are related to the limited amount of harmonics included in the solution. When the number of harmonic terms increase, the system of equations written in matrix form can be ill-conditioned and the solution becomes inaccurate. This problem has been reduced in our calculations by including proper scaling of machine model dimensions in radial direction for all regions

$$
r_{\text {scal }}=r_{\text {real }} 10^{x c}
$$

for a given real radii of the studied machine (Table I), new scaled radii are given by equation (75).

Limiting the number of harmonics for limiting computational time will lead to inaccurate field solutions, especially for PM machines where airgap length is small, PM subregion with high height and small thickness and high number of subregions with different spatial frequencies. For the studied parallel double excitation PM machine, the number of subregions is equal to 50. To obtain an accurate magnetic field solution, $x c=1.45$ with the number of harmonics 250 for $n$ and 80 for $m$ and $k$. An optimum number of harmonics and scaling factor with limiting computational time can be found with using for example, different harmonics for all of the 8 regions. Of course, the number of harmonics in the airgap and PM should be high but the number of harmonics in the non magnetic region VI can be small.

Figs. 7 to 14 show a comparison between analytical and FEM open circuit, armature reaction and on load magnetic field distribution results in the middle of the airgap of parallel double excitation PM machine accounting for tooth tips and real structure of polar piece. The results are in excellent 
agreement. This type of PM machines has a small magnet arc to pole pitch ratio $(0.2)$ and a very high height $(29.8 \mathrm{~mm})$ in comparison with radial surface mounted and inset PM machines which present a small PM height and high width. Analytical method based on subdomain model give a very good accuracy even when PM subregion is deep. In Figs. 7 to 12 , radial and tangential flux density in the middle of the airgap is given for 3 conditions: Permanent magnets alone; stator currents alone and rotor excitation currents alone. The radial and tangential flux density distribution for on-load condition is shown in Figs. 13 and 14. The machine is fed by $120^{\circ}$ rectangular stator current and we consider the permanent magnets and rotor DC current acting together. Figs. 15 and 16 show the flux lines obtained by FEM due to rotor excitation current and stator currents. We can observe that all analytical results are in very good agreement with those obtained by FEM. In Figs. 17 to 20, we show radial and tangential flux densities for on load condition in the cases where the hybrid PM machine has stator and rotor slots fed by current and PM does not exist and only stator slots are fed and PM are considered. Those two cases represent the contribution of permanent magnet and rotor excitation current to magnetic field on load. In control process of magnetic field in the air gap, current excitation can be set to zero to decrease magnetic field. It is important to note here that the results are obtained for a recoil relative permeability of permanent magnet equal to unity, but the method take into account the effect of non unity permeability. Figs. 21 to 26 show open circuit, armature reaction and on load radial and tangential flux density in the middle of airgap in integer slot winding Spoke-Type PM machine accounting for tooth tips and real structure of polar piece. All analytical results are in very good agreement with those obtained by FEM, which confirm the accuracy of analytical subdomain model in predictions of this type of permanent magnet machines even when a PM subregion is very deep with small thickness and with taking into account tooth tips and real structure of polar piece. Fig. 27 shows magnetic field on load obtained by FEM in Spoke-Type PM machine.

TABLE I

Parameters OF Studied Parallel Double Excitation AND SPOKE-TyPe PERMANENT-MAGNET MOTOR

\begin{tabular}{lcc}
\hline \hline Parameter & Symbol & $\begin{array}{c}\text { Value and } \\
\text { unit }\end{array}$ \\
\hline Magnet remanence (Ferrite) & $B_{r}$ & $0.4 \mathrm{~T}$ \\
Relative recoil permeability of magnet & $\mu_{r}$ & 1 \\
Number of conductors per stator slot & $N_{c}$ & 12 \\
Peak phase current & $I_{m}$ & $12.5 \mathrm{~A}$ \\
DC excitation current & $I_{f}$ & $5 \mathrm{~A}$ \\
Number of conductors per rotor slot & $N_{f}$ & 10 \\
Number of stator slots & $Q_{s}$ & 12 \\
Stator slot opening width & $c$ & $14^{\circ}$ \\
Rotor slot opening width & $c_{r}$ & $14^{\circ}$ \\
Number of pole pairs & $p$ & 2 \\
Number of rotor excitation slots & $N_{r}$ & 8 \\
External radius of rotor slot & $r_{0}$ & $40.8 \mathrm{~mm}$ \\
Internal radius of rotor slot & $r_{1}$ & $27.8 \mathrm{~mm}$ \\
External radius of magnet & $r_{2}$ & $40 \mathrm{~mm}$ \\
Internal radius of stator slot & $r_{3}$ & $49 \mathrm{~mm}$ \\
External radius of stator slot & $r_{4}$ & $58 \mathrm{~mm}$ \\
Rotor slot opening width & $d r$ & $8^{\circ}$ \\
Magnet slot opening width & $b$ & $12^{\circ}$ \\
Stator slot opening width & $d$ & $8^{\circ}$ \\
Radius of the external stator surface & $R_{o}$ & $70 \mathrm{~mm}$ \\
\end{tabular}

Radius of the stator outer surface

Radius of the rotor inner surface at the magnet surface

Radius of the rotor inner surface at the magnet bottom

Air-gap length

Height of a magnet

Height of stator slot

Stack length

Magnet arc to pole pitch ratio Rotor speed

wrm $157 \mathrm{rd} / \mathrm{s}$

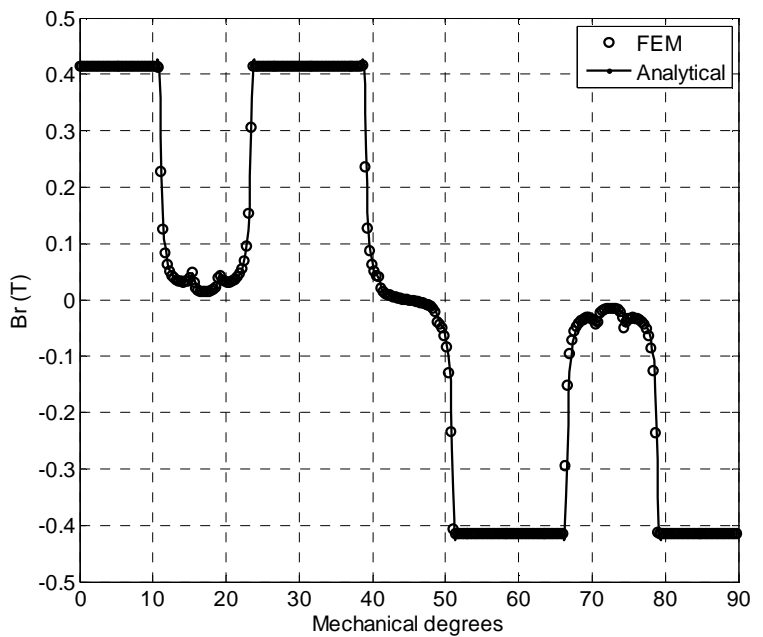

Fig. 7. Radial flux density due to permanent magnet alone in parallel double excitation PM machine

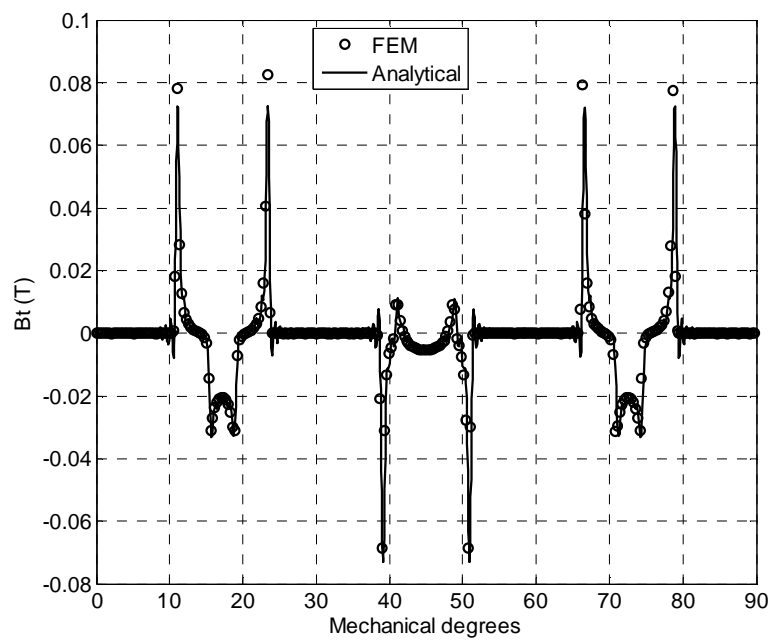

Fig. 8. Tangential flux density due to permanent magnet alone in parallel double excitation PM machine 


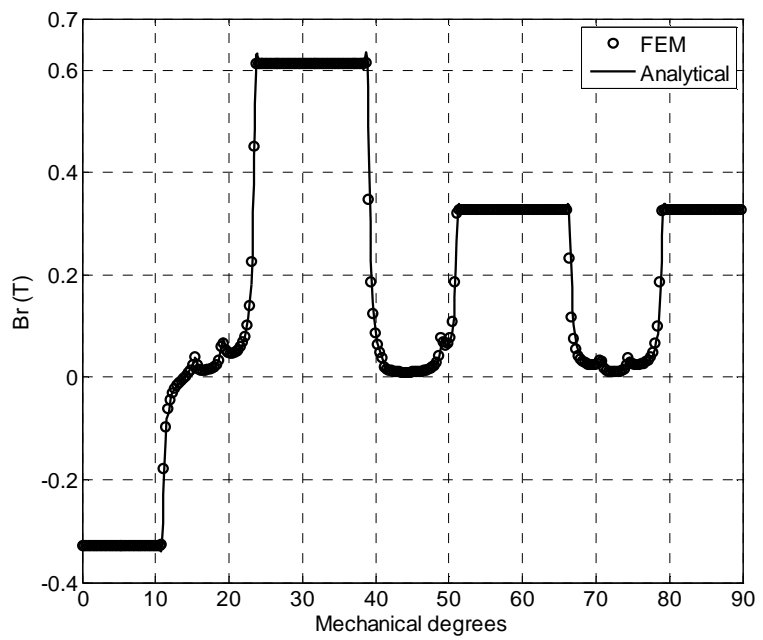

Fig. 9. Radial flux density due to stator current alone in parallel double excitation PM machine

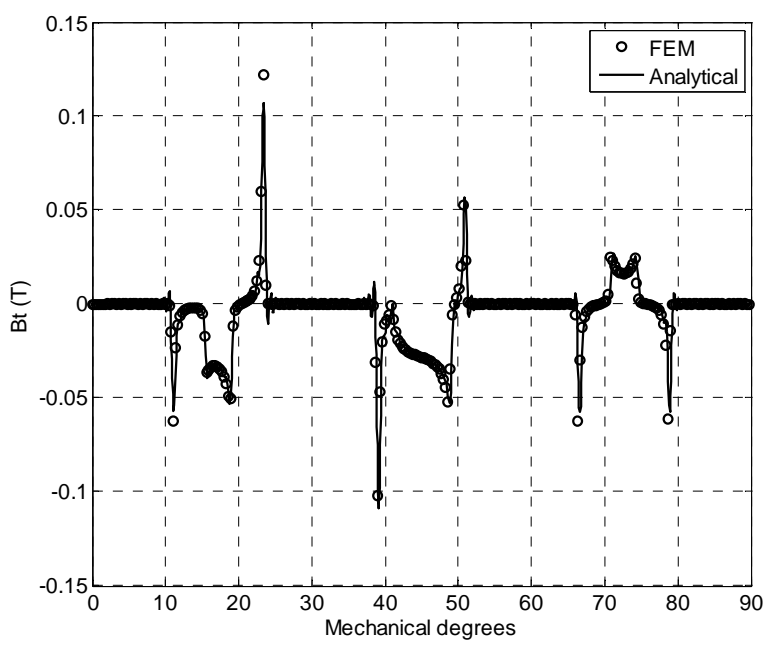

Fig. 10. Tangential flux density due to stator current alone in parallel double excitation PM machine

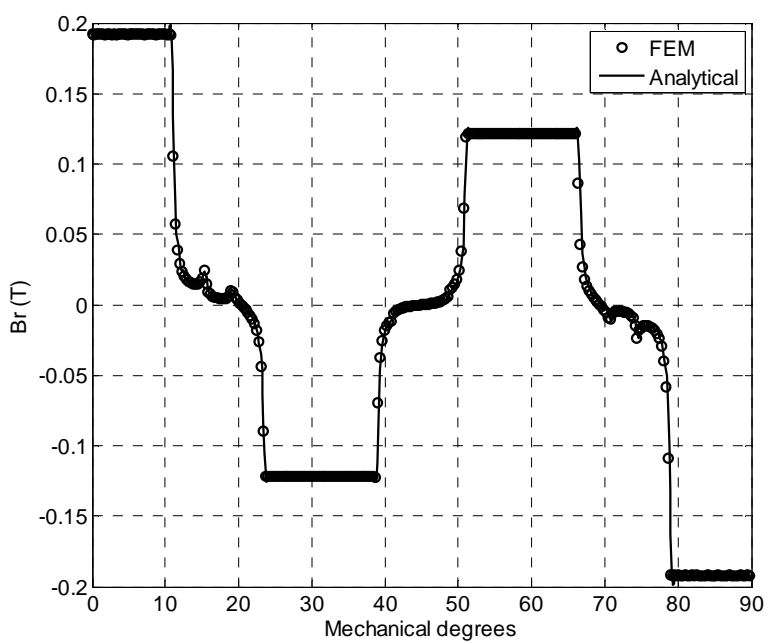

Fig. 11. Radial flux density due to rotor excitation current alone in parallel double excitation PM machine

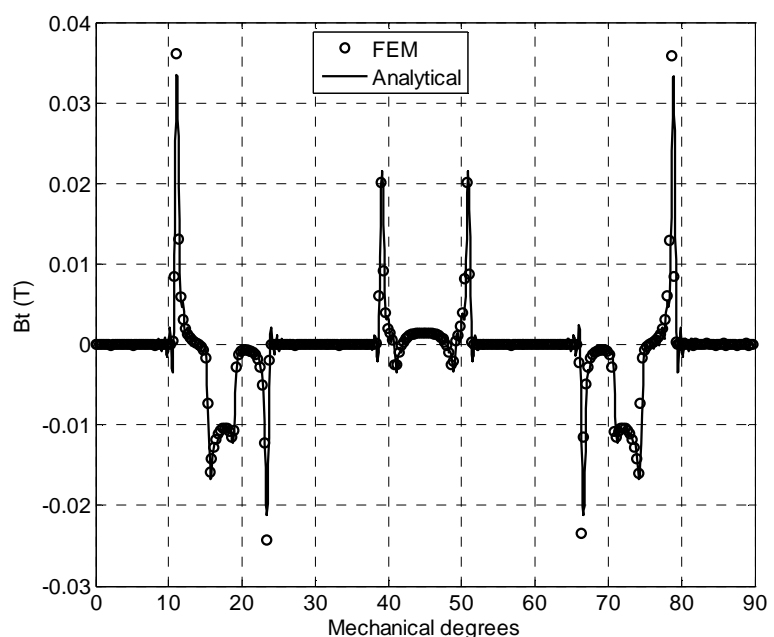

Fig. 12. Tangential flux density due to rotor excitation current alone in parallel double excitation PM machine

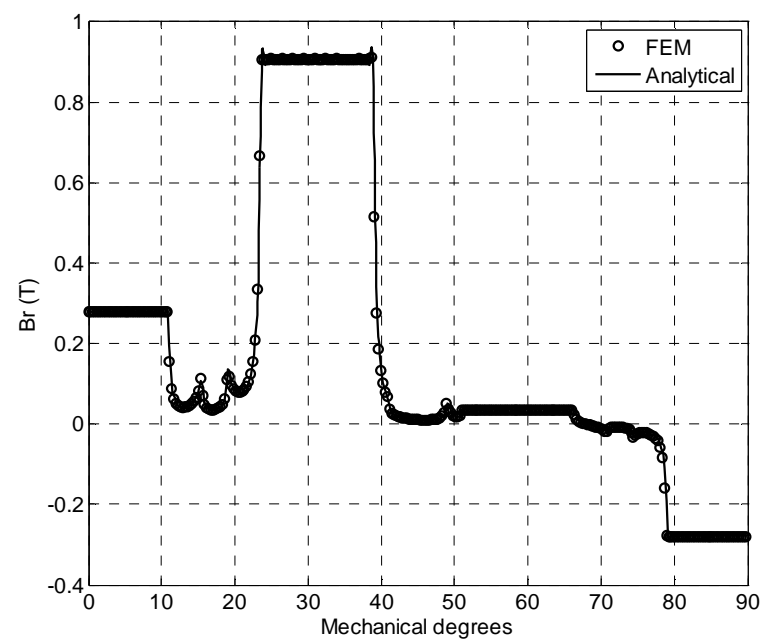

Fig. 13. Radial flux density on load in parallel double excitation PM machine

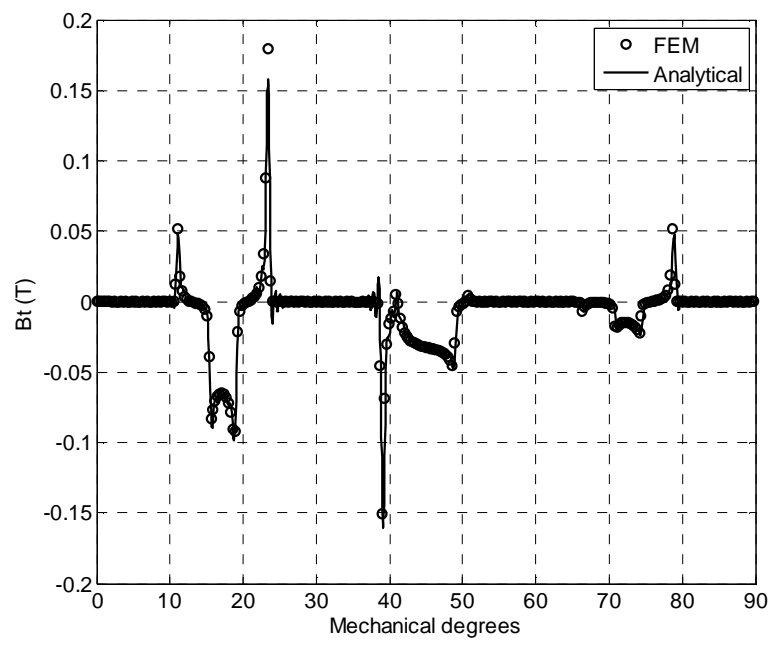

Fig. 14. Tangential flux density on load in parallel double excitation PM machine 


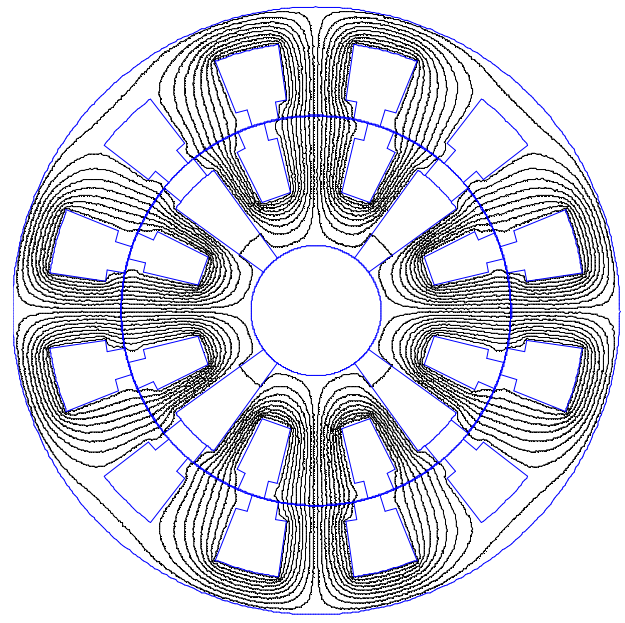

Fig. 15. Magnetic field due to rotor double excitation current alone in parallel double excitation PM machine

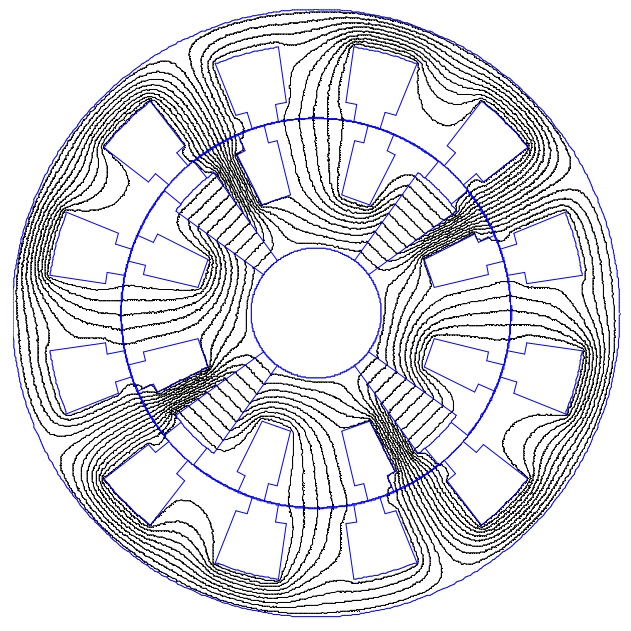

Fig. 16. Magnetic field on load in parallel double excitation PM machine

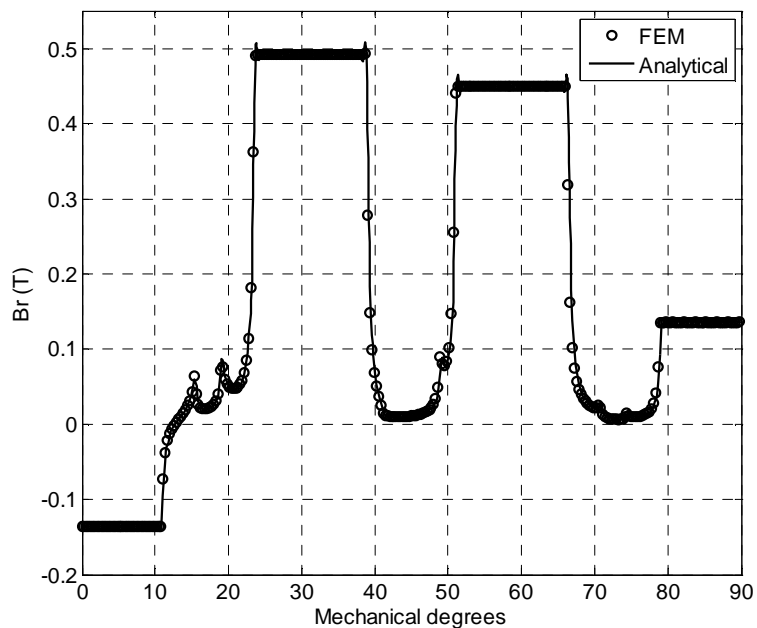

Fig. 17. Radial flux density due to rotor excitation and stator currents (on load) in parallel double excitation PM machine

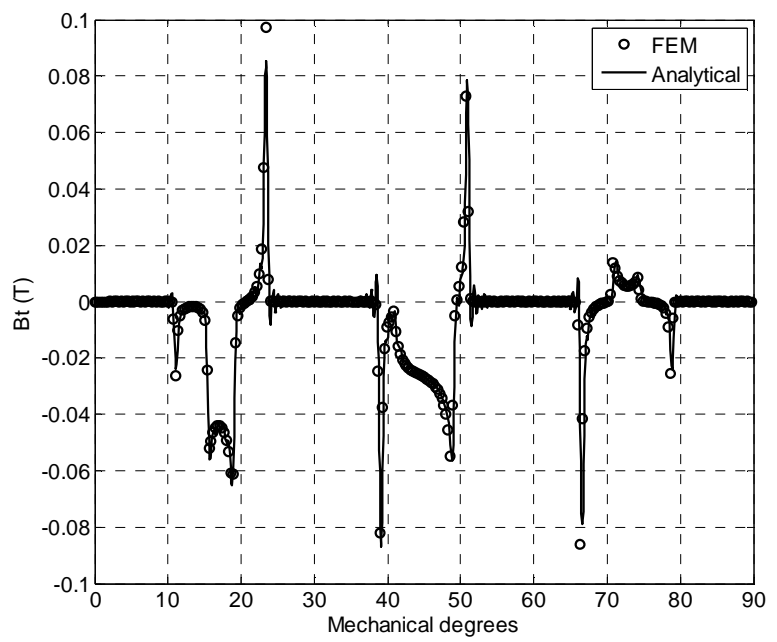

Fig. 18. Tangential flux density due to rotor excitation and stator currents (on load) in parallel double excitation PM machine

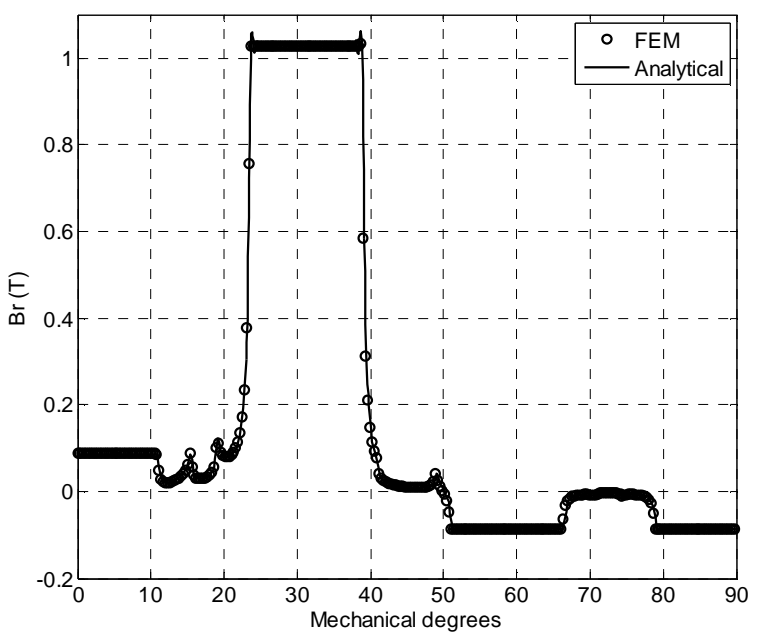

Fig. 19. Radial flux density due to permanent magnet and stator currents (on load) in parallel double excitation PM machine

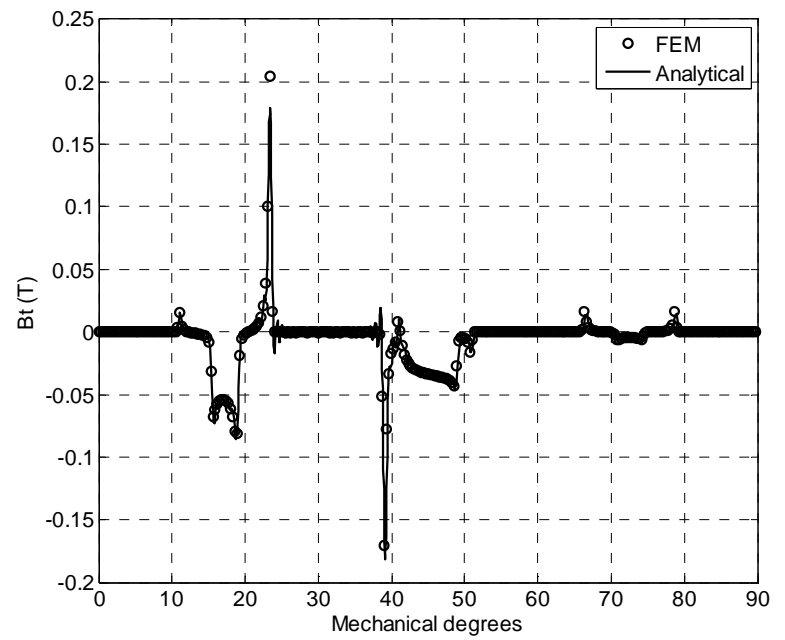

Fig. 20. Tangential flux density due to permanent magnet and stator currents (on load) in parallel double excitation PM machine 


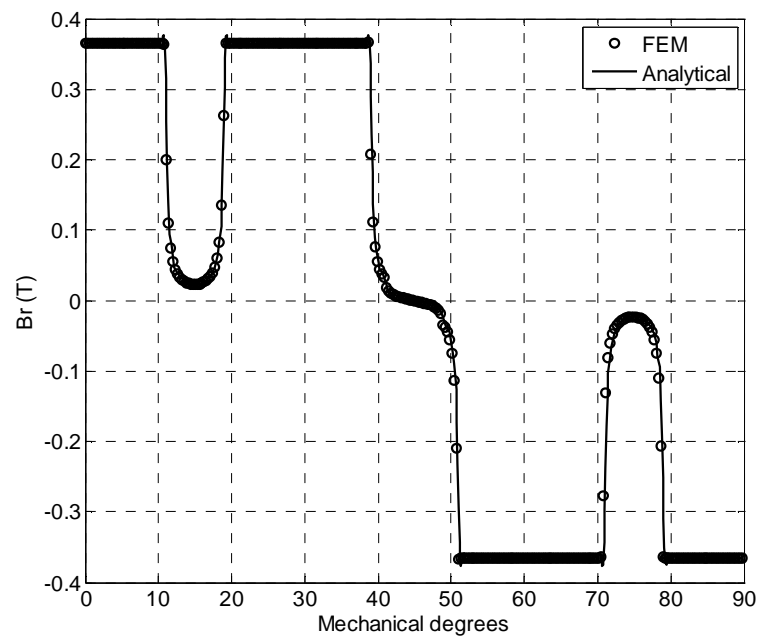

Fig. 21. Radial flux density due to permanent magnet alone in

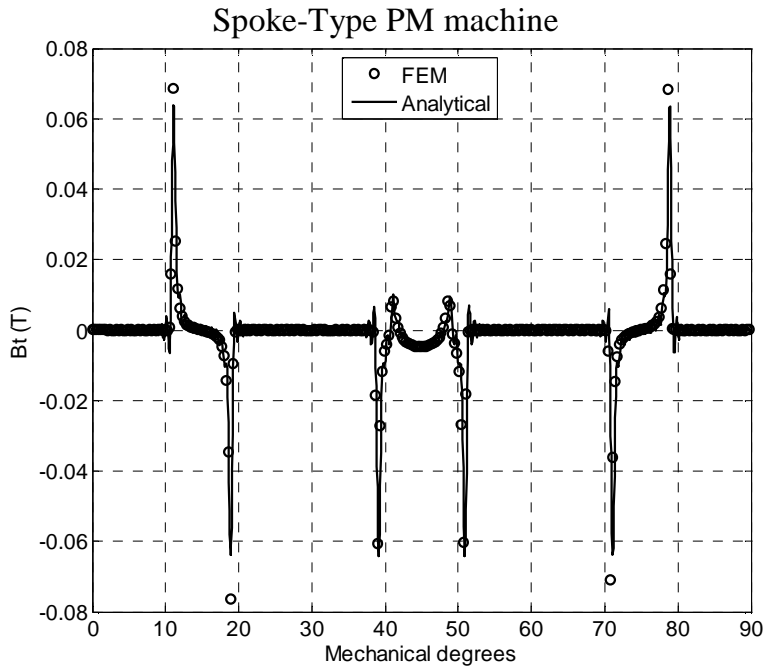

Fig. 22. Tangential flux density due to permanent magnet alone in Spoke-Type PM machine

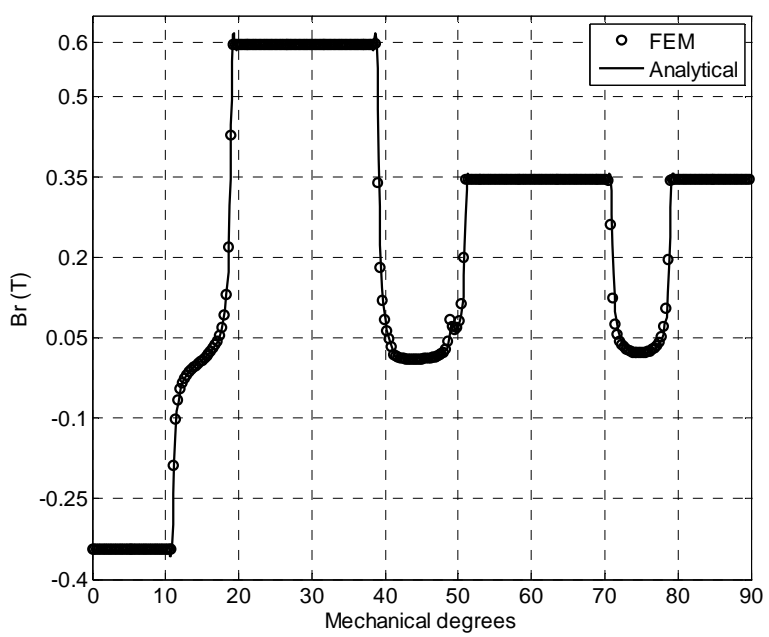

Fig. 23. Radial flux density due to stator current alone in Spoke-Type PM machine

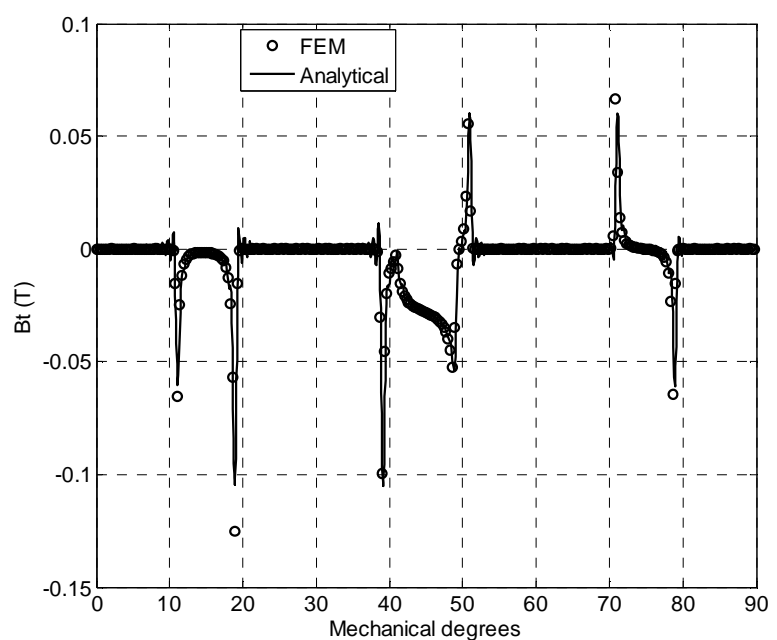

Fig. 24. Tangential flux density due to stator current alone in Spoke-Type PM machine

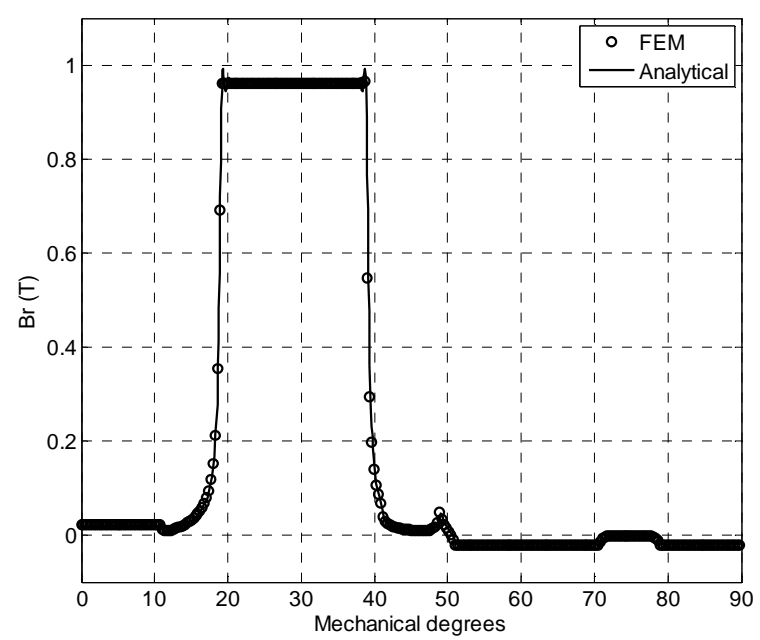

Fig. 25. Radial flux density on load in Spoke-Type PM machine

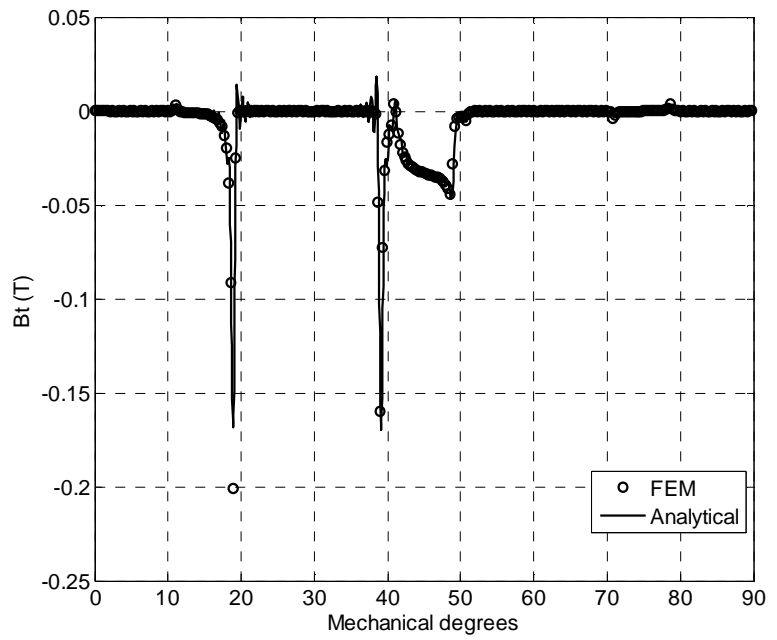

Fig. 26. Tangential flux density on load in Spoke-Type PM machine 


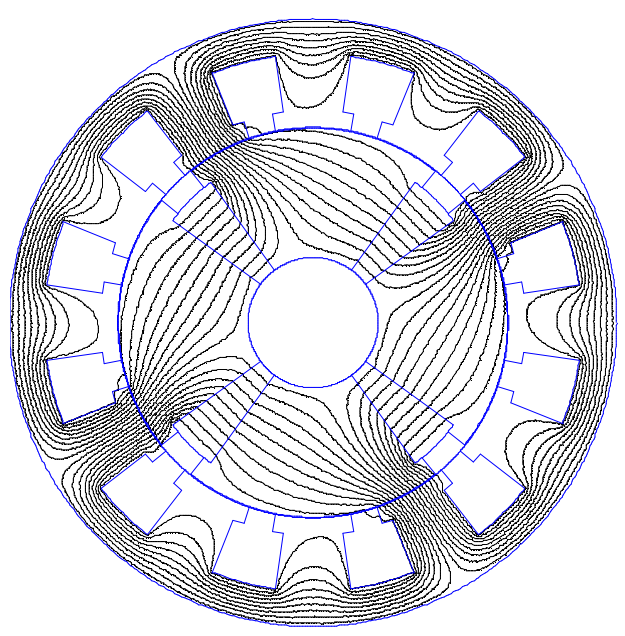

Fig. 27. Magnetic field on load in Spoke-Type PM machine

\section{CONCLUSION}

In this paper, we have proposed an improved analytical subdomain model for predicting open circuit, armature reaction and on-load magnetic field distribution in integer stator slot distributed winding parallel double excitation and Spoke-Type permanent magnet machines. The proposed model takes into account the rotor and stator slots tooth tips and the shape of polar pieces. The whole domain is divided into eight subregions for parallel double excitation motor and six subregions for Spoke-Type permanent magnet motor, stator semi-closed slots, rotor semi-closed slots, airgap, buried tangential permanent magnet and non magnetic region under magnet. Poisson's and Laplace's equations are solved analytically using the method of separation of variables. Analytical results are in excellent agreement with the ones obtained by FEM. From these results, we have shown the accuracy of analytical subdomain model to predict magnetic field accounting for rotor and stator tooth tips and shape of polar piece, even when PM subregion is deep with small thickness.

\section{APPENDIX}

Fourier series coefficients of general solution in different regions of parallel double excitation and Spoke-Type permanent magnet machines are determined by resolution of a system of equations as seen above. Some of those equations are detailed as follows.

From equation (46), we get

$$
\begin{aligned}
& A 5_{j, 0}+A 6_{j, 0} \ln \left(R_{r}\right)-M_{j} \mu_{0} R_{r} \\
= & \frac{1}{a} \sum_{n=1}^{\infty}\left(A 7_{n} R_{r}^{n p}\right) \int_{g_{j}-\frac{a}{2}}^{g_{j}+\frac{a}{2}} \sin (n p \theta) d \theta \\
+ & \frac{1}{a} \sum_{n=1}^{\infty}\left(A 9_{n} R_{r}^{n p}\right) \int_{g_{j}-\frac{a}{2}}^{g_{j}+\frac{a}{2}} \cos (n p \theta) d \theta
\end{aligned}
$$

$$
\begin{aligned}
& A 5_{j, m} R_{r}^{-\frac{m \pi}{a}}+A 6_{j, m} R_{r}^{\frac{m \pi}{a}} \\
& =\frac{2}{a} \sum_{n=1}^{\infty}\left(A 7_{n} R_{r}^{n p}\right) \text {. } \\
& \int_{g_{j}-\frac{a}{2}}^{g_{j}+\frac{a}{2}} \sin (n p \theta) \cos \left(\frac{m \pi}{a}\left(\theta-g_{j}+\frac{a}{2}\right)\right) d \theta \\
& +\frac{2}{a} \sum_{n=1}^{\infty}\left(A 9_{n} R_{r}^{n p}\right) . \\
& \int_{g_{j}-\frac{a}{2}}^{g_{j}+\frac{a}{2}} \cos (n p \theta) \cos \left(\frac{m \pi}{a}\left(\theta-g_{j}+\frac{a}{2}\right)\right) d \theta \\
& \text { where } \int_{g_{j}-\frac{a}{2}}^{g_{j}+\frac{a}{2}} \sin (n p \theta) \cos \left(\frac{m \pi}{a}\left(\theta-g_{j}+\frac{a}{2}\right)\right) d \theta \\
& =\frac{n p a^{2}\left(-1+(-1)^{m}\right) \cos \left(\frac{n p a}{2}\right) \cos \left(n p g_{j}\right)}{m^{2} \pi^{2}-p^{2} n^{2} a^{2}} \\
& +\frac{n p a^{2}\left(-1-(-1)^{m}\right) \sin \left(\frac{n p a}{2}\right) \sin \left(n p g_{j}\right)}{m^{2} \pi^{2}-p^{2} n^{2} a^{2}}
\end{aligned}
$$

for $m^{2} \pi^{2}-p^{2} n^{2} a^{2} \neq 0$, and

$$
\begin{aligned}
& =\frac{\cos \left(-n p g_{j}+\frac{n p a}{2}\right)}{4 n p}-\frac{\cos \left(n p g_{j}+\frac{3 n p a}{2}\right)}{4 n p} \\
& -\frac{a \sin \left(-n p g_{j}+\frac{n p a}{2}\right)}{2} \text { for } m^{2} \pi^{2}-p^{2} n^{2} a^{2}=0
\end{aligned}
$$$$
\text { and } \int_{g_{j}-\frac{a}{2}}^{g_{j}+\frac{a}{2}} \cos (n p \theta) \cos \left(\frac{m \pi}{a}\left(\theta-g_{j}+\frac{a}{2}\right)\right) d \theta
$$

$$
\begin{aligned}
& =-\frac{n p a^{2}\left(1+(-1)^{m}\right) \sin \left(\frac{n p a}{2}\right) \cos \left(n p g_{j}\right)}{m^{2} \pi^{2}-p^{2} n^{2} a^{2}} \\
& -\frac{n p a^{2}\left(-1+(-1)^{m}\right) \sin \left(n p g_{j}\right) \cos \left(\frac{n p a}{2}\right)}{m^{2} \pi^{2}-p^{2} n^{2} a^{2}}
\end{aligned}
$$

for $m^{2} \pi^{2}-p^{2} n^{2} a^{2} \neq 0$ and 
$=\frac{a \cos \left(-n p g_{j}+\frac{n p a}{2}\right)}{2}+\frac{\sin \left(-n p g_{j}+\frac{n p a}{2}\right)}{4 n p}$

$+\frac{\sin \left(n p g_{j}+\frac{3 n p a}{2}\right)}{4 n p}$ for $m^{2} \pi^{2}-p^{2} n^{2} a^{2}=0$.

From equation (48), we have:

$\left(\frac{n p}{\mu_{0}}\right)\left(-A 7_{n} R_{r}^{n p-1}\right)$

$=\frac{1}{\pi \mu_{0} \mu_{r}} \sum_{j=1}^{2 p} \sum_{m=1}^{\infty}\left(\frac{m \pi}{a} A 5_{j, m} R_{r}^{-\frac{m \pi}{a}-1}-\frac{m \pi}{a} A 6_{j, m} R_{r}^{\frac{m \pi}{a}-1}\right)$.

$\int_{g_{j}-\frac{a}{2}}^{g_{j}+\frac{a}{2}} \sin (n p \theta) \cos \left(\frac{m \pi}{a}\left(\theta-g_{j}+\frac{a}{2}\right)\right) d \theta$

$-\left(\frac{1}{\pi \mu_{0} \mu_{r}}\right) \sum_{j=1}^{2 p} \frac{A 6_{j, 0}}{R_{r}} \int_{g_{j}-\frac{a}{2}}^{g_{j}+\frac{a}{2}} \sin (n p \theta) d \theta$

Equation (49) gives

$\left(\frac{n p}{\mu_{0}}\right)\left(-A 9_{n} R_{r}^{n p-1}\right)$

$=\frac{1}{\pi \mu_{0} \mu_{r}} \sum_{j=1}^{2 p} \sum_{m=1}^{\infty}\left(\frac{m \pi}{a} A 5_{j, m} R_{r}^{-\frac{m \pi}{a}-1}-\frac{m \pi}{a} A 6_{j, m} R_{r}^{\frac{m \pi}{a}-1}\right)$.

$\int_{g_{j}-\frac{a}{2}}^{g_{j}+\frac{a}{2}} \cos (n p \theta) \cos \left(\frac{m \pi}{a}\left(\theta-g_{j}+\frac{a}{2}\right)\right) d \theta$

$-\left(\frac{1}{\pi \mu_{0} \mu_{r}}\right) \sum_{j=1}^{2 p} \frac{A 6_{j, 0}}{R_{r}} \int_{g_{j}-\frac{a}{2}}^{g_{j}+\frac{a}{2}} \cos (n p \theta) d \theta$

Equation (50) gives

$$
\begin{aligned}
& A 13_{j, 0}+A 14_{j, 0} \ln \left(r_{2}\right) \\
& =A 5_{j, 0}+A 6_{j, 0} \ln \left(r_{2}\right)-M_{j} \mu_{0} r_{2} \\
& +\frac{1}{b} \sum_{m=1}^{\infty}\left(A 5_{j, m} r_{2}^{-\frac{m \pi}{a}}+A 6_{j, m} r_{2}^{\frac{m \pi}{a}}\right) . \\
& \int_{g_{j}-\frac{b}{2}}^{g_{j}+\frac{b}{2}} \cos \left(\frac{m \pi}{a}\left(\theta-g_{j}+\frac{a}{2}\right)\right) d \theta
\end{aligned}
$$

$$
\begin{aligned}
& \text { where } \int_{g_{j}-\frac{b}{2}}^{g_{j}+\frac{b}{2}} \cos \left(\frac{m \pi}{a}\left(\theta-g_{j}+\frac{a}{2}\right)\right) d \theta \\
& =\frac{2 a \sin \left(\frac{\pi m p}{2 a}\right) \cos \left(\frac{m \pi}{2}\right)}{\pi m}
\end{aligned}
$$

From equation (51), we have

$$
A 13_{j, k} r_{2}^{-\frac{k \pi}{b}}+A 14_{j, k} r_{2}^{\frac{k \pi}{b}}
$$

$$
=\frac{2}{b} \sum_{m=1}^{\infty}\left(A 5_{j, m} r_{2}^{-\frac{m \pi}{a}}+A 6_{j, m} r_{2}^{\frac{m \pi}{a}}\right) \text {. }
$$

$\int_{g_{j}-\frac{b}{2}}^{g_{j}+\frac{b}{2}} \cos \left(\frac{m \pi}{a}\left(\theta-g_{j}+\frac{a}{2}\right)\right) \cos \left(\frac{k \pi}{b}\left(\theta-g_{j}+\frac{b}{2}\right)\right) d \theta$

$$
+\frac{2}{b}\left(A 5_{j, 0}+A 6_{j, 0} \ln \left(r_{2}\right)-M_{j} \mu_{0} r_{2}\right) \text {. }
$$$$
\int_{g_{j}-\frac{b}{2}}^{g_{j}+\frac{b}{2}} \cos \left(\frac{k \pi}{b}\left(\theta-g_{j}+\frac{b}{2}\right)\right) d \theta
$$

$$
\text { where } \int_{g_{j}-\frac{b}{2}}^{g_{j}+\frac{b}{2}} \cos \left(\frac{k \pi}{b}\left(\theta-g_{j}+\frac{b}{2}\right)\right) d \theta=0, \text { and }
$$$$
\int_{g_{j}-\frac{b}{2}}^{g_{j}+\frac{b}{2}} \cos \left(\frac{m \pi}{a}\left(\theta-g_{j}+\frac{a}{2}\right)\right) \cos \left(\frac{k \pi}{b}\left(\theta-g_{j}+\frac{b}{2}\right)\right) d \theta
$$$$
=-\frac{m b^{2} a\left(1+(-1)^{k}\right) \sin \left(\frac{\pi m b}{2 a}\right) \cos \left(\frac{m \pi}{2}\right)}{\pi\left(-m^{2} b^{2}+k^{2} a^{2}\right)}
$$$$
-\frac{m b^{2} a\left(-1+(-1)^{k}\right) \sin \left(\frac{m \pi}{2}\right) \cos \left(\frac{\pi m b}{2 a}\right)}{\pi\left(-m^{2} b^{2}+k^{2} a^{2}\right)}
$$

for $-m^{2} b^{2}+k^{2} a^{2} \neq 0$ and

$=\frac{1}{4 \pi k}\left(2 b k \pi \cos \left(\frac{-b k \pi+k \pi a}{2 b}\right)\right)$

$+\frac{1}{4 k \pi}\left(-b \sin \left(\frac{-b k \pi+k a \pi}{2 b}\right)+b \sin \left(\frac{3 b k \pi+k a \pi}{2 b}\right)\right)$

for $-m^{2} b^{2}+k^{2} a^{2}=0$.

Development of equation (52) is reduced to

$$
-\frac{A 6_{j, 0}}{r_{2}} \frac{1}{\mu_{0} \mu_{r}}=-\frac{A 14_{j, 0} b}{a \mu_{0} r_{2}}
$$


From equation (53), we have

$$
\begin{aligned}
& \frac{1}{\mu_{0} \mu_{r}} \frac{m \pi}{a}\left(A 5_{j, m} r_{2}^{-\frac{m \pi}{a}-1}-A 6_{j, m} r_{2}^{\frac{m \pi}{a}-1}\right) \\
& =\frac{2}{a \mu_{0}}\left(-\frac{A 14_{j, 0}}{r_{2}}\right)_{g_{j}-\frac{b}{2}}^{g_{j}+\frac{b}{2}} \cos \left(\frac{m \pi}{a}\left(\theta-g_{j}+\frac{a}{2}\right)\right) d \theta \\
& -\frac{2}{a \mu_{0}} \sum_{k=1}^{\infty} \frac{k \pi}{b}\left(-A 13_{j, k} r_{2}^{-\frac{k \pi}{b}-1}+A 14_{j, k} r_{2}{ }^{\frac{k \pi}{b}-1}\right) . \\
& \int_{g_{j}+\frac{b}{2}} \cos \left(\frac{k \pi}{b}\left(\theta-g_{j}+\frac{b}{2}\right)\right) \cos \left(\frac{m \pi}{a}\left(\theta-g_{j}+\frac{a}{2}\right)\right) d \theta
\end{aligned}
$$

From equation (54), we have

$$
A 13_{j, 0}+A 14_{j, 0} \ln \left(R_{m}\right)
$$$$
=\frac{1}{b} \sum_{n=1}^{\infty}\left(A 1_{n} R_{m}^{n p}+A 2_{n} R_{m}^{-n p}\right) \int_{g_{j}-\frac{b}{2}}^{g_{j}+\frac{b}{2}} \sin (n p \theta) d \theta
$$$$
+\frac{1}{b} \sum_{n=1}^{\infty}\left(A 3_{n} R_{m}^{n p}+A 4_{n} R_{m}^{-n p}\right) \int_{g_{j}-\frac{b}{2}}^{g_{j}+\frac{b}{2}} \cos (n p \theta) d \theta
$$

Equation (55) development gives

$$
\begin{aligned}
& A 13_{j, k} R_{m}^{-\frac{k \pi}{b}}+A 14_{j, k} R_{m}^{\frac{k \pi}{b}} \\
& =\frac{2}{b} \sum_{n=1}^{\infty}\left(A 1_{n} R_{m}^{n p}+A 2_{n} R_{m}^{-n p}\right) . \\
& g_{j}+\frac{b}{2} \sin (n p \theta) \cos \left(\frac{k \pi}{b}\left(\theta-g_{j}+\frac{b}{2}\right)\right) d \theta \\
& g_{g_{j}-\frac{b}{2}} \\
& +\frac{2}{b} \sum_{n=1}^{\infty}\left(A 3_{n} R_{m}^{n p}+A 4_{n} R_{m}^{-n p}\right) . \\
& g_{j}+\frac{b}{2} \\
& \int_{g_{j}-\frac{b}{2}}^{2} \cos (n p \theta) \cos \left(\frac{k \pi}{b}\left(\theta-g_{j}+\frac{b}{2}\right)\right) d \theta
\end{aligned}
$$

From equation (56), we have

$$
\begin{aligned}
& A 15_{i r, 0}+A 16_{i r, 0} \ln \left(R_{m}\right) \\
& =\frac{1}{b} \sum_{n=1}^{\infty}\left(A 1_{n} R_{m}^{n p}+A 2_{n} R_{m}^{-n p}\right) \cdot \int_{\beta_{i r}-\frac{d r}{2}}^{\beta_{i r}+\frac{d r}{2}} \sin (n p \theta) d \theta \\
& +\frac{1}{b} \sum_{n=1}^{\infty}\left(A 3_{n} R_{m}^{n p}+A 4_{n} R_{m}^{-n p}\right) \cdot \int_{\beta_{i r}-\frac{d r}{2}}^{\beta_{i r}+\frac{d r}{2}} \cos (n p \theta) d \theta
\end{aligned}
$$

From equation (57), we have

$$
\begin{aligned}
& A 15_{i r, k} R_{m}^{-\frac{k \pi}{d r}}+A 16_{i r, k} R_{m}^{\frac{k \pi}{d r}} \\
= & \frac{2}{d r} \sum_{n=1}^{\infty}\left(A 1_{n} R_{m}^{n p}+A 2_{n} R_{m}^{-n p}\right) . \\
\beta_{i r}+\frac{d r}{2} & \int_{\beta_{i r}-\frac{d r}{2}} \sin (n p \theta) \cos \left(\frac{k \pi}{d r}\left(\theta-\beta_{i r}+\frac{d r}{2}\right)\right) d \theta \\
+ & \frac{2}{d r} \sum_{n=1}^{\infty}\left(A 3_{n} R_{m}^{n p}+A 4_{n} R_{m}^{-n p}\right) .
\end{aligned}
$$

$\int_{\beta_{i r}-\frac{d r}{2}}^{\beta_{i r}+\frac{d r}{2}} \cos (n p \theta) \cos \left(\frac{k \pi}{d r}\left(\theta-\beta_{i r}+\frac{d r}{2}\right)\right) d \theta$

Development of equation (58) gives

$$
\begin{aligned}
& \frac{n p}{\mu_{0}}\left(-A 1_{n} R_{m}^{n p-1}+A 2_{n} R_{m}^{-n p-1}\right) \\
= & \frac{1}{\pi \mu_{0}} \sum_{j=1}^{2 p} \sum_{k=1}^{\infty} \frac{k \pi}{b}\left(A 13_{j, k} R_{m}^{-\frac{k \pi}{b}-1}-A 14_{j, k} R_{m}^{\frac{k \pi}{b}-1}\right) . \\
& \int_{g_{j}-\frac{b}{2}}^{g_{j}+\frac{b}{2}} \sin (n p \theta) \cos \left(\frac{k \pi}{b}\left(\theta-g_{j}+\frac{b}{2}\right)\right) d \theta
\end{aligned}
$$$$
-\sum_{j=1}^{2 p} \frac{A 14_{j, 0}}{\pi R_{m} \mu_{0}} \int_{g_{j}-\frac{b}{2}}^{g_{j}+\frac{b}{2}} \sin (n p \theta) d \theta
$$$$
+\sum_{i r=1}^{N_{r}} \sum_{k=1}^{\infty} \frac{k \pi}{d r}\left(A 15_{i r, k} R_{m}^{-\frac{k \pi}{d r}-1}-A 16_{i r, k} R_{m}^{\frac{k \pi}{d r}-1}\right) .
$$$$
\int_{\beta_{i r}-\frac{d r}{2}}^{\beta_{i r}+\frac{d r}{2}} \sin (n p \theta) \cos \left(\frac{k \pi}{d r}\left(\theta-\beta_{i r}+\frac{d r}{2}\right)\right) d \theta
$$$$
-\sum_{i r=1}^{N_{r}}\left(\frac{A 16_{i r, 0}}{\pi \mu_{0} R_{m}}\right)_{\beta_{i r}-\frac{d r}{2}}^{\beta_{i r}+\frac{d r}{2}} \sin (n p \theta) d \theta
$$

From equation (59), we have

$$
\begin{aligned}
& \frac{n p}{\mu_{0}}\left(-A 3_{n} R_{m}^{n p-1}+A 4_{n} R_{m}^{-n p-1}\right) \\
& =\frac{1}{\pi \mu_{0}} \sum_{j=1}^{2 p} \sum_{k=1}^{\infty} \frac{k \pi}{b}\left(A 13_{j, k} R_{m}^{-\frac{k \pi}{b}-1}-A 14_{j, k} R_{m}^{\frac{k \pi}{b}-1}\right) . \\
& \int_{g_{j}+\frac{b}{2}}^{2} \cos (n p \theta) \cos \left(\frac{k \pi}{b}\left(\theta-g_{j}+\frac{b}{2}\right)\right) d \theta
\end{aligned}
$$


$-\sum_{j=1}^{2 p} \frac{A 14_{j, 0}}{\pi R_{m} \mu_{0}} \int_{g_{j}-\frac{b}{2}}^{g_{j}+\frac{b}{2}} \cos (n p \theta) d \theta$

$+\sum_{i r=1}^{N_{r}} \sum_{k=1}^{\infty} \frac{k \pi}{d r}\left(A 15_{i r, k} R_{m}^{-\frac{k \pi}{d r}-1}-A 16_{i r, k} R_{m}^{\frac{k \pi}{d r}-1}\right)$.

$\int_{\beta_{i r}-\frac{d r}{2}}^{\beta_{i r}+\frac{d r}{2}} \cos (n p \theta) \cos \left(\frac{k \pi}{d r}\left(\theta-\beta_{i r}+\frac{d r}{2}\right)\right) d \theta$

$-\sum_{i r=1}^{N_{r}}\left(\frac{A 16_{i r, 0}}{\pi \mu_{0} R_{m}}\right)_{\beta_{i r}-\frac{d r}{2}}^{\beta_{i r}+\frac{d r}{2}} \cos (n p \theta) d \theta$

Equation (60) development is

$$
\begin{aligned}
& A 11_{i, 0}+A 12_{i, 0} \ln \left(R_{s}\right) \\
& =\frac{1}{d} \sum_{n=1}^{\infty}\left(A 1_{n} R_{s}^{n p}+A 2_{n} R_{s}^{-n p}\right) \int_{\alpha_{i}-\frac{d}{2}}^{\alpha_{i}+\frac{d}{2}} \sin (n p \theta) d \theta \\
& +\frac{1}{d} \sum_{n=1}^{\infty}\left(A 3_{n} R_{s}^{n p}+A 4_{n} R_{s}^{-n p}\right) \int_{\alpha_{i}-\frac{d}{2}}^{\alpha_{i}+\frac{d}{2}} \cos (n p \theta) d \theta
\end{aligned}
$$

From equation (61), we have

$$
\begin{aligned}
& A 11_{i, k} R_{s}^{-\frac{k \pi}{d}}+A 12_{i, k} R_{s}^{\frac{k \pi}{d}} \\
& =\frac{2}{d} \sum_{n=1}^{\infty}\left(A 1_{n} R_{s}^{n p}+A 2_{n} R_{s}^{-n p}\right) . \\
& \int_{\alpha_{i}+\frac{d}{2}}^{2} \cos \left(\frac{k \pi}{d}\left(\theta-\alpha_{i}+\frac{d}{2}\right)\right) \sin (n p \theta) d \theta \\
& \alpha_{i}-\frac{d}{2} \\
& +\frac{2}{d} \sum_{n=1}^{\infty}\left(A 3_{n} R_{s}^{n p}+A 4_{n} R_{s}^{-n p}\right) . \\
& \alpha_{i}+\frac{d}{2} \\
& \int_{\alpha_{i}-\frac{d}{2}}^{2} \cos \left(\frac{k \pi}{d}\left(\theta-\alpha_{i}+\frac{d}{2}\right)\right) \cos (n p \theta) d \theta
\end{aligned}
$$

From equation (62), we have

$$
\begin{aligned}
& \frac{n p}{\mu_{0}}\left(-A 1_{n} R_{s}^{n p-1}+A 2_{n} R_{s}^{-n p-1}\right) \\
& =\frac{1}{\pi \mu_{0}} \sum_{i=1}^{Q_{s}} \sum_{k=1}^{\infty}-\frac{k \pi}{d}\left(-A 11_{i, k} R_{s}^{-\frac{k \pi}{d}-1}+A 12_{i, k} R_{s}^{\frac{k \pi}{d}-1}\right) .
\end{aligned}
$$

$\int_{\alpha_{i}-\frac{d}{2}}^{\alpha_{i}+\frac{d}{2}} \cos \left(\frac{k \pi}{d}\left(\theta-\alpha_{i}+\frac{d}{2}\right)\right) \sin (n p \theta) d \theta$

$-\sum_{i=1}^{Q_{s}} \frac{A 12_{i, 0}}{\pi R_{s} \mu_{0}} \int_{\alpha_{i}-\frac{d}{2}}^{\alpha_{i}+\frac{d}{2}} \sin (n p \theta) d \theta$

From equation (63), we have

$\frac{n p}{\mu_{0}}\left(-A 3_{n} R_{s}^{n p-1}+A 4_{n} R_{s}^{-n p-1}\right)$

$=\frac{1}{\pi \mu_{0}} \sum_{i=1}^{Q_{s}} \sum_{k=1}^{\infty}-\frac{k \pi}{d}\left(-A 11_{i, k} R_{s}^{-\frac{k \pi}{d}-1}+A 12_{i, k} R_{s}^{\frac{k \pi}{d}-1}\right)$.

(A-15)

$\int_{\alpha_{i}-\frac{d}{2}}^{\alpha_{i}+\frac{d}{2}} \cos \left(\frac{k \pi}{d}\left(\theta-\alpha_{i}+\frac{d}{2}\right)\right) \cos (n p \theta) d \theta$

$-\sum_{i=1}^{Q_{s}} \frac{A 12_{i, 0}}{\pi R_{s} \mu_{0}} \int_{\alpha_{i}-\frac{d}{2}}^{\alpha_{i}+\frac{d}{2}} \cos (n p \theta) d \theta$

From equation (64), we have

$$
\begin{aligned}
& A 11_{i, 0}+A 12_{i, 0} \ln \left(r_{3}\right) \\
& =C_{i, 0}+\frac{1}{2} \mu_{0} J_{i} r_{4}^{2} \ln \left(r_{3}\right)-\frac{1}{4} \mu_{0} J_{i} r_{3}^{2} \\
& +\frac{1}{d} \sum_{m=1}^{\infty} C_{i, m}\left(\left(\frac{r_{3}}{r_{4}}\right)^{\frac{m \pi}{c}}-\left(\frac{r_{3}}{r_{4}}\right)^{-\frac{m \pi}{c}}\right) . \\
& \int_{\alpha_{i}+\frac{d}{2}} \cos \left(\frac{m \pi}{c}\left(\theta-\alpha_{i}+\frac{c}{2}\right)\right) d \theta
\end{aligned}
$$

From equation (65), we have

$$
A 11_{i, k} r_{3}^{-\frac{k \pi}{d}}+A 12_{i, k} r_{3}^{\frac{k \pi}{d}}
$$

$=\frac{2}{d}\left(C_{i, 0}+\frac{1}{2} \mu_{0} J_{i} r_{4}^{2} \ln \left(r_{3}\right)-\frac{1}{4} \mu_{0} J_{i} r_{3}^{2}\right)$.

$\int_{\alpha_{i}-\frac{d}{2}}^{\alpha_{i}+\frac{d}{2}} \cos \left(\frac{k \pi}{d}\left(\theta-\alpha_{i}+\frac{d}{2}\right)\right) d \theta$

$+\frac{2}{d} \sum_{m=1}^{\infty} C_{i, m}\left(\left(\frac{r_{3}}{r_{4}}\right)^{\frac{m \pi}{c}}-\left(\frac{r_{3}}{r_{4}}\right)^{-\frac{m \pi}{c}}\right)$. 
$\int_{\alpha_{i}-\frac{d}{2}}^{\alpha_{i}+\frac{d}{2}} \cos \left(\frac{m \pi}{c}\left(\theta-\alpha_{i}+\frac{c}{2}\right)\right) \cos \left(\frac{k \pi}{d}\left(\theta-\alpha_{i}+\frac{d}{2}\right)\right) d \theta$

Equation (66) is reduced to

$\frac{1}{\mu_{0}}\left(-\frac{1}{2} \frac{\mu_{0} J_{i} r_{4}^{2}}{r_{3}}+\frac{1}{2} \mu_{0} J_{i} r_{3}\right)=-\frac{A 12_{i, 0} d}{c \mu_{0} r_{3}}$

From equation (67), we have

$$
\begin{aligned}
& -\frac{C_{i, m}}{\mu_{0}}\left(\frac{m \pi}{c r_{3}}\right)\left(\left(\frac{r_{3}}{r_{4}}\right)^{\frac{m \pi}{c}}+\left(\frac{r_{3}}{r_{4}}\right)^{-\frac{m \pi}{c}} \quad\right. \text { (A-22) } \\
& =\frac{2}{c \mu_{0}}\left(-\frac{A 12_{i, 0}}{r_{3}}\right)_{\alpha_{i}-\frac{d}{2}}^{\alpha_{i}+\frac{d}{2}} \cos \left(\frac{m \pi}{c}\left(\theta-\alpha_{i}+\frac{c}{2}\right)\right) d \theta \\
& +\frac{2}{c \mu_{0}} \sum_{k=1}^{\infty} \frac{k \pi}{d}\left(A 11_{i, k} r_{3}^{-\frac{k \pi}{d}-1}-A 12_{i, k} r_{3}^{\frac{k \pi}{d}-1}\right) . \\
& \int_{i}+\frac{d}{2} \\
& \int_{\alpha_{i}-\frac{d}{2}} \cos \left(\frac{k \pi}{d}\left(\theta-\alpha_{i}+\frac{d}{2}\right)\right) \cos \left(\frac{m \pi}{c}\left(\theta-\alpha_{i}+\frac{c}{2}\right)\right) d \theta
\end{aligned}
$$

From equation (68), we have

$$
\begin{aligned}
& A 15_{i r, 0}+A 16_{i r, 0} \ln \left(r_{0}\right) \\
& =C 1_{i r, 0}+\frac{1}{2} \mu_{0} J_{f i r} r_{1}^{2} \ln \left(r_{0}\right)-\frac{1}{4} \mu_{0} J_{f i r} r_{0}^{2} \\
& +\frac{1}{d r} \sum_{m=1}^{\infty} C 1_{i r, m}\left(\left(\frac{r_{0}}{r_{1}}\right)^{\frac{m \pi}{c_{r}}}-\left(\frac{r_{0}}{r_{1}}\right)^{-\frac{m \pi}{c_{r}}}\right) . \\
& \int_{\beta_{i r}-\frac{d r}{2}}^{\beta_{i}} \cos \left(\frac{m \pi}{c r}\left(\theta-\beta_{i r}+\frac{c r}{2}\right)\right) d \theta
\end{aligned}
$$

From equation (69), we have

$$
\begin{aligned}
& A 15_{i r, k} r_{0}^{-\frac{k \pi}{d r}}+A 16_{i r, k} r_{0}^{\frac{k \pi}{d r}} \\
& =\frac{2}{d r}\left(C 1_{i r, 0}+\frac{1}{2} \mu_{0} J_{f i r} r_{1}^{2} \ln \left(r_{0}\right)-\frac{1}{4} \mu_{0} J_{f i r} r_{0}^{2}\right) . \\
& \beta_{i r}+\frac{d r}{2} \cos \left(\frac{k \pi}{d r}\left(\theta-\beta_{i r}+\frac{d r}{2}\right)\right) d \theta \\
& +\frac{2}{d r} \sum_{m=1}^{\infty} C 1_{i r, m}\left(\left(\frac{r_{0}}{r_{1}}\right)^{\frac{m \pi}{c r}}-\left(\frac{r_{0}}{r_{1}}\right)^{-\frac{m \pi}{c r}}\right) .
\end{aligned}
$$
Shaogang and Z. Anming "State of the Art of Hybrid Excitation Permanent Magnet Synchronous Machines", Electrical Machines and Systems (ICEMS), 2010 International Conference on, pp. 1004-1009.

[3] Y. Amara, S. Hlioui, R. Belfkira, G. Barakat and M. Gabsi "Comparison of Open Circuit Flux Control Capability of a Series Double Excitation Machine and a Parallel Double Excitation Machine", IEEE Trans. Vehicular Technology., Vol. PP, No. 99, pp. 1, 2011.

[4] A. D. Akemakou, S. K. Phounsombat, "Electrical machine with double excitation especially a motor vehicle alternator," Nov. 14, 2000, US Patent 6,147,429.

[5] H. Bali, Y. Amara, G. Barakat, R. Ibtiouen and M. Gabsi "Analytical Modeling of Open Circuit Magnetic Field in Wound Field and Series Double Excitation Synchronous Machines", IEEE Trans. Magnetics., Vol. 46, No. 10, pp. 3802-3815, October 2010.

[6] R. L. Owen, Z. Q. Zhu and G. W. Jewell "Hybrid-Excited FluxSwitching Permanent-Magnet Machines With Iron Flux Bridges", IEEE Trans. Magnetics., Vol. 46, No. 6, pp. 1726-1729, June 2010.

[7] Z. Zhang, Y. Tao and Y. Yan "Investigation of a New Topology of Hybrid Excitation Doubly Salient Brushless DC Generator", IEEE Trans. Industrial Electronics, Vol. PP, No. 99, pp. 1-8, 2011. 
[8] E. Sulaiman, T. Kosaka and N. Matsui "Design Optimization of 12Slot10Pole Hybrid Excitation Flux Switching Synchronous Machine with $0.4 \mathrm{~kg}$ Permanent Magnet for Hybrid Electric Vehicles", Power Electronics and ECCE Asia (ICPE \& ECCE), 2011 IEEE 8th International Conference on, pp. 1913-1920, 2011.

[9] K. Y. Hwang, J. H. Jo and B. I. Kwon "A Study on Optimal Pole Design of Spoke-Type IPMSM With Concentrated Winding for Reducing the Torque Ripple by Experiment Design Method", IEEE Trans. Magnetics., Vol. 45, No. 10, pp. 4712-4715, October 2009.

[10] Yul-kyu Son, Kyu-yun Hwang and Byung-il Kwon "Maximum Torque Control for Optimal Design to Reduce Cogging Torque in Spoke Type Interior Permanent Magnet Synchronous Motor", Energy Conversion Congress and Exposition (ECCE), 2010 IEEE, pp. 3654-3658.

[11] Hao Qian, Hong Guo, Zhi Huang and Zhiyong Wu "Multi-Domain Analysis of Tangential-set Permanent Magnet Synchronous Generator", Electrical Machines and Systems (ICEMS), 2010 International Conference on, pp. 1773-1776.

[12] D. Lin, P. Zhou and Z. J. Cendes "Analytical Prediction of Cogging Torque in Spoke Type Permanent Magnet Motors", Electrical Machines, 2008. ICEM 2008. $18^{\text {th }}$ International Conference on, pp. 1-5.

[13] Huaishu Li, Hao Xiong, Yuqi Rang, Qiang Wu, Guangwei Meng and Libing Zhou "Analytical Calculation of Inductance Parameter of Tangentially Magnetized PM Motor Based on Basic Air-gap Magnetic Field", Artificial Intelligence, Management Science and Electronic Commerce (AIMSEC), $20112^{\text {nd }}$ International Conference on, pp. 37763781.

[14] K. Boughrara "Modélisation Analytique et Numérique des Performances Electromagnétiques des Moteurs Synchrones à Aimants Permanents", Doctorat d'Etat thesis, Ecole Nationale Polytechnique, Algiers, December 2008.

[15] T. Lubin, S. Mezani and A. Rezzoug "2-D Exact Analytical Model for Surface-Mounted Permanent-Magnet Motors With Semi-Closed Slots", IEEE Trans. Magnetics., Vol. 47, No. 2, pp. 479-492, February 2011.

[16] Z. Q. Zhu, L. J. Wu and Z. P. Xia "An Accurate Subdomain Model for Magnetic Field Computation in Slotted Surface-Mounted PermanentMagnet Machines", IEEE Trans. Magnetics., Vol. 46, No. 4, pp. 11001115, April 2010.

[17] L. J. Wu, Z. Q. Zhu, D. Staton, M. Popescu and D. Hawkins "An Improved Subdomain Model for Predicting Magnetic Field of SurfaceMounted Permanent Magnet Machines Accounting for Tooth-Tips", IEEE Trans. Magnetics., Vol. 47, No. 6, pp. 1693-1704, June 2011.

[18] L. Jian, K. T. Chau, Y. Gong, C. Yu and W. Li "Analytical Calculation of Magnetic Field in Surface-Inset Permanent Magnet Motors", IEEE Trans. Magnetics., Vol. 45, No. 10, pp. 4688-4691, October 2009.

[19] L. Jian, G. Xu, C. C. Mi, K. T. Chau, C. C. Chan "Analytical Method for Magnetic Field Calculation in a Low-Speed Permanent-Magnet Harmonic Machine", IEEE Trans. Energy Conversion., Vol. 26, No. 3 pp. 862-870, September 2011

[20] Y. Amara, G. Brakat and P. Reghem "Armature Reaction Magnetic Field of Tubular Linear Surface-Inset Permanent-Magnet Machines", IEEE Trans. Magnetics., Vol. 47, No. 4, pp. 805-811, April 2011.

[21] Y. Amara and G. Brakat "Analytical Modeling of Magnetic Field I Surface Mounted Permanent-Magnet Tubular Linear Machines", IEEE Trans. Magnetics., Vol. 46, No. 11, pp. 3870-3884, November 2010.

[22] B. L. J. Gysen, K. J. Meessen, J. J. H. Paulides and E. A. Lomonova "General Formulation of the Electromagnetic Field Distribution in Machines and Devices Using Fourier Analysis", IEEE Trans. Magnetics., Vol. 46, No. 1, pp. 39-51, January 2010.

[23] B. L. J. Gysen, E. Ilhan, K. J. Meessen, J. J. H. Paulides and E. A. Lomonova "Modeling of Flux Switching Permanent Magnet Machines With Fourier Analysis", IEEE Trans. Magnetics., Vol. 46, No. 6, pp. 1499-1502, June 2010.

[24] J. Wang, D. Howe and G. W. Jewell "Analysis and Design Optimization of an Improved Axially Magnetized Tubular Permanent-Magnet Machine”, IEEE Trans. Magnetics., Vol. 19, No. 2, pp. 289-295, June 2004

[25] K. Boughrara, D. Zarko, R. Ibtiouen, O. Touhami and A. Rezzoug "Magnetic Field Analysis of Inset and Surface Mounted Permanent Magnet Synchronous Motors using Schwarz-Christoffel Transformation ", IEEE Trans. Magnetics., Vol. 45, No. 8, pp. 3166-3178, August 2009.

[26] T. Lubin, S. Mezani and A. Rezzoug "Exact Analytical Method for Magnetic Field Computation in the Air Gap of Cylindrical Electrical Machines Considering Slotting Effects", IEEE Trans. Magnetics., Vol 46, No. 4, pp. 1092-1099, April 2010.
[27] D. C. Meeker, Finite Element Method Magnetics. ver. 4.2 (1 Apr. 2009 Build) [Online]. Available: http://www.femm.info

Kamel Boughrara was born in Algiers, Algeria, in 1969. He received the engineer diploma from the University of Sciences and Technology Houari Boumediene and the magister degree from Ecole Nationale Polytechnique, Algiers, Algeria, in 1994 and 1997 respectively, and the Doctorat d'Etat degree from Ecole Nationale Polytechnique, Algeria, in 2008. He is currently a Lecturer at the Université de Khemis Miliana, Algeria, at the Laboratoire de l'Energie et des systèmes intelligents. His interests include modeling and control of electrical machines.

Rachid Ibtiouen was born in Algeria in 1955. He received the Ph.D. degree in electrical engineering from the Ecole Nationale Polytechnique ENP Algiers and from the Institut National Polytechnique de Lorraine (INPL), France) in 1993. From 1988 to 1993 , he integrated the Groupe de Recherche en Electrotechnique et Electronique de Nancy (GREEN). Between 1986 till 1999, he was three times a Head of Electrical Engineering Department in the ENP. From 2005 till 2008, he was a Deputy Director in charge of Studies in the ENP Algiers. From 1993 till 2011, He is an expert member of the committee of evaluation of the university projects of research (CNEPRU), with the ministry of the Algerian Higher education. He is currently a Professor and Associated Director of Research with the Ecole Nationale Polytechnique. Since January 2005 until today, He was the Director of the Research Laboratory of Electrical Engineering (LRE) in ENP. His fields of scientific interest include the modeling electric systems and drives, particularly electrical machines.

Thierry Lubin was born in Sedan, France, in 1970. He received the M.S. degree from the University of Paris 6, France in 1994 and the Ph.D. degree from the University Henri Poincaré, Nancy, France, in 2003.

$\mathrm{He}$ is currently a lecturer of Electrical Engineering at the University of Nancy at the Groupe de Recherche en Electrotechnique et Electronique de Nancy. His interests include modeling and control of electrical machines and applied superconductivity in electrical devices. 